ARTICLE

Received 28 Feb 2014 | Accepted 21 Jul 2014 | Published 21 Aug $2014 \quad$ DOI: 10.1038/ncomms5742

\title{
Enhanced synapse remodelling as a common phenotype in mouse models of autism
}

Masaaki Isshiki ${ }^{1, \star}$, Shinji Tanaka ${ }^{1, \star}$, Toshihiko Kuriu ${ }^{2}$, Katsuhiko Tabuchi ${ }^{3,4}$, Toru Takumi ${ }^{5,6}$ \& Shigeo Okabe ${ }^{1}$

Developmental deficits in neuronal connectivity are considered to be present in patients with autism spectrum disorders (ASDs). Here we examine this possibility by using in vivo spine imaging in the early postnatal cortex of ASD mouse models. Spines are classified by the presence of either the excitatory postsynaptic marker PSD-95 or the inhibitory postsynaptic marker gephyrin. ASD mouse models show consistent upregulation in the dynamics of PSD-95-positive spines, which may subsequently contribute to stable synaptic connectivity. In contrast, spines receiving inputs from the thalamus, detected by the presence of gephyrin clusters, are larger, highly stable and unaffected in ASD mouse models. Importantly, two distinct mouse models, human 15q11-13 duplication and neuroligin-3 R451C point mutation, show highly similar phenotypes in spine dynamics. This selective impairment in dynamics of PSD-95-positive spines receiving intracortical projections may be a core component of early pathological changes and be a potential target of early intervention.

\footnotetext{
${ }^{1}$ Department of Cellular Neurobiology, Graduate School of Medicine, the University of Tokyo, 7-3-1 Hongo, Bunkyo-ku, Tokyo 113-0033, Japan. ${ }^{2}$ Department of Neurophysiology, Kagawa School of Pharmaceutical Sciences, Tokushima Bunri University, Kagawa 769-2193, Japan. ${ }^{3}$ Department of Molecular and Cellular Physiology, Shinshu University School of Medicine, 3-1-1 Asahi, Matsumoto, Nagano 390-8621, Japan. ${ }^{4}$ PRESTO, Japan Science and Technology Agency (JST), 4-1-8 Honcho Kawaguchi, Saitama 332-0012, Japan. ${ }^{5}$ RIKEN Brain Science Institute, 2-1 Hirosawa, Wako, Saitama 351-0198, Japan. ${ }^{6}$ CREST, Japan Science and Technology Agency (JST), 4-1-8 Honcho Kawaguchi, Saitama 332-0012, Japan. * These authors contributed equally to this work. Correspondence and requests for materials should be addressed to S.O. (email: okabe@m.u-tokyo.ac.jp).
} 
C ortical dysfunction is considered to underlie deficits in social behaviours and communication in autism spectrum disorders (ASDs) ${ }^{1}$. Symptoms of ASDs manifest during early development. Because of recent advances in early screening, ASDs can be reliably diagnosed by 3 years of age. Neuropathological studies of patients with ASD indicated relatively small changes in brain architecture and few signs of neurodegeneration ${ }^{2}$. The early onset of this disease and small pathological changes in the brain of patients with ASD led to the hypothesis that impairment in synapse formation, neuronal connectivity and circuit stabilization may explain the pathogenesis of $\mathrm{ASDs}^{3-5}$.

In agreement with the abovementioned concept, genetic studies of copy number variants (CNVs) and gene mutations have supported the hypothesis that non-syndromic ASDs can be caused by structural or sequence variations in genes related to synapse development, including genes encoding cell adhesion and scaffolding molecules ${ }^{6-8}$. Furthermore, mouse molecular genetics confirmed an impairment of synaptic functions, including GABA signalling and long-term potentiation/depression, in multiple ASD mouse models ${ }^{8-15}$. Although these studies provide strong evidence that supports a causal relationship between ASD-related gene mutations and synapse pathology, little is known about synapse formation and remodelling in the developing neocortex of these mouse models. As ASD symptoms manifest during the early stages of cortical development, detection of impairment in synapse formation, stability and remodelling will provide essential information in identifying core circuit-level defects at the onset of the disease.

A wide heterogeneity exists among patients with ASDs, and defining the core characteristics of this disease is very difficult from only the presentation of symptoms. To define the core characteristics of ASDs, neurobiological studies of mouse models and subgrouping based on genetic evidences are essential. Research on several diseases comorbid with autism has provided models linking defects in intracellular signalling with dysfunctions of synapses. Rett syndrome, fragile $\mathrm{X}$ syndrome and tuberous sclerosis complex exhibit comorbidity with ASDs, and their corresponding mouse models were established and extensively studied ${ }^{16-19}$. Rescue experiments using these syndromic ASD mouse models successfully identified key molecular pathways involved in the pathology of neural circuits and behavioural deficits ${ }^{9-11,20-22}$. However, most ASD cases are non-syndromic, and recent genetic studies of non-syndromic ASDs identified a large number of candidates with rare genetic variants ${ }^{6,7}$. These studies revealed the important roles of both rare mutations and CNVs in the aetiology of ASDs. Interestingly, rare variants in genes of postsynaptic neuroligin-3 and -4 (NLGN 3 and 4), as well as their presynaptic binding partner neurexin-1 $(N R X N 1)$ have been linked with $\mathrm{ASDs}^{8}$. These findings suggest that the interaction between pre- and postsynaptic membranes has important roles in non-syndromic ASDs. Among a variety of CNVs detected in ASDs, duplication of 15q11-13 has been most frequently reported, suggesting that the dosage of one or more genes in this chromosomal region is critical for normal brain function $^{23}$. To extract common neurodevelopmental defects in non-syndromic ASDs, comparison of mouse models that mimic rare mutations in NLGN and chromosomal duplication in 15q11-13 may provide indispensable information. Therefore, we characterized the following two types of non-syndromic ASD mouse models: patDp/ + mice ${ }^{24}$, which mimic human chromosome 15q11-13 duplication, and NLG R451C mice ${ }^{15}$, which are engineered to have a rare missense mutation of Nlgn3 similar to that of patients with ASD in this study.

We performed in vivo two-photon time-lapse imaging in developing layer $2 / 3$ pyramidal neurons expressing green fluorescent protein (GFP)-tagged synaptic markers and the red fluorescent protein DsRed2. Dendritic spines with PSD-95 clusters showed enhanced turnover in both patDp/ + and NLG $\mathrm{R} 451 \mathrm{C}$ mice. Spines receiving input from the thalamus that are detected by the presence of gephyrin clusters were larger, highly stable and unaffected in both ASD mouse models. Thus, spines containing postsynaptic densities (PSDs) and receiving intracortical projections are consistently affected in the developing cortex of two non-syndromic ASD mouse models. This selective impairment in synaptic dynamics may be a core component of early pathological changes at the onset of ASDs and a possible target of early intervention for the disease.

\section{Results}

Dynamics of spines classified by postsynaptic markers. The primary somatosensory barrel cortex (SSC) of rodents has been extensively studied as a model system of experience-dependent development of neural circuits ${ }^{25,26}$. In this analysis, we first focused on synapses formed onto layer $2 / 3$ pyramidal neurons in the SSC. These neurons are major integrators of sensory information and receive multiple inputs, including those from layer 4 spiny stellate cells, other layer $2 / 3$ pyramidal cells and the thalamus (thalamocortical axons (TCAs) $)^{27}$. Previous studies indicated a rapid postnatal increase of synapse density in the SSC $^{28-30}$, but precise developmental profiles of spines in layer $2 / 3$ pyramidal neurons have not been obtained. Therefore, we measured the spine density of layer $2 / 3$ pyramidal neurons along the course of postnatal development and found a rapid increase in spine density until postnatal week 3 and its stabilization thereafter (Fig. 1a,b).

To further illustrate the underlying mechanism of spine density increase, we next measured spine turnover in vivo (Fig. 1c). We performed in vivo two-photon imaging, mainly with a transcranial thinned-skull window, in this study ${ }^{31,32}$. In some experiments with young animals, we applied the open-skull technique $^{33}$ (refer to Methods for details). In vivo two-photon imaging revealed that the gain of spines exceeded elimination at postnatal week 2 , but it was downregulated and balanced with their elimination at postnatal week 3 . This timing was consistent with the developmental profile of total spine density (Fig. 1b,c), suggesting that the balance of gain and loss is an important determinant of the increase in postnatal spine density. By setting a specific time window at postnatal week 3 in the initial analysis of the ASD mouse model, we aimed to detect any impairment in the regulatory mechanisms of spine remodelling. In this time window, spine density already reached the level of mature SSC, but active remodelling of spines still occurs, and both functional and structural plasticity is robust ${ }^{25,31,32}$.

We used two types of markers, PSD-95 tagged with GFP (PSD-95-GFP) and gephyrin tagged with GFP (gephyrin-GFP), for classification of spines imaged in vivo. Expression of these markers did not affect spine turnover monitored by in vivo imaging of DsRed-filled dendrites (Supplementary Fig. 1a). PSD-95-GFP clusters are a reliable marker for the postsynaptic structure $^{34-36}$. Therefore, spines containing PSD-95-GFP (PSD-95-GFP- $(+)$ spines) can be classified as more differentiated spines. Consistent with this idea, PSD-95-GFP- $(-)$ spines were a minor fraction compared with PSD-95-GFP- $(+)$ spines, and their volumes were significantly smaller (Fig. 1d). PSD-95GFP- $(-)$ spines were distinct from dendritic filopodia based on their differences in lifetimes (refer to Methods). The grouping of spines with or without PSD-95-GFP clusters along postnatal development revealed selective increases of PSD-95-GFP- $(+)$ spines from postnatal day (PND) 7 to 22, without significant changes in the density of PSD-95-GFP- $(-)$ spines (Fig. 1b). 
a

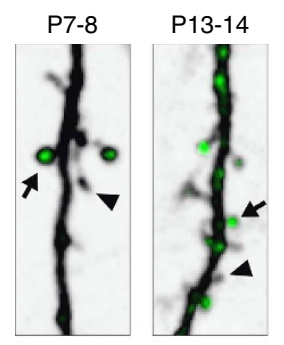

C

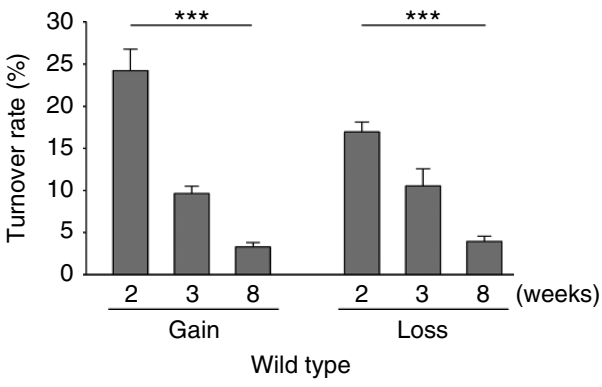

b

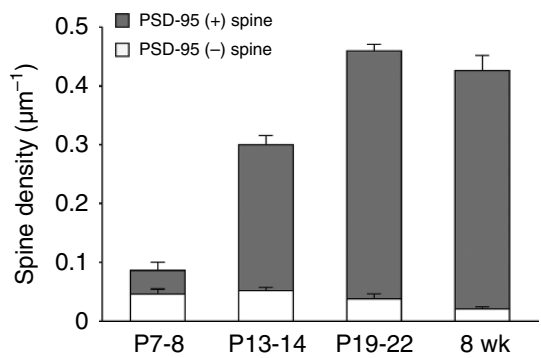

d

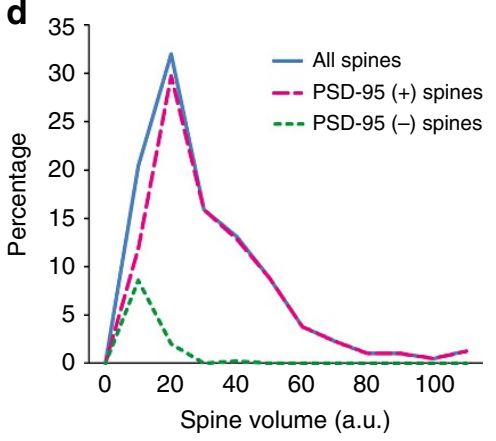

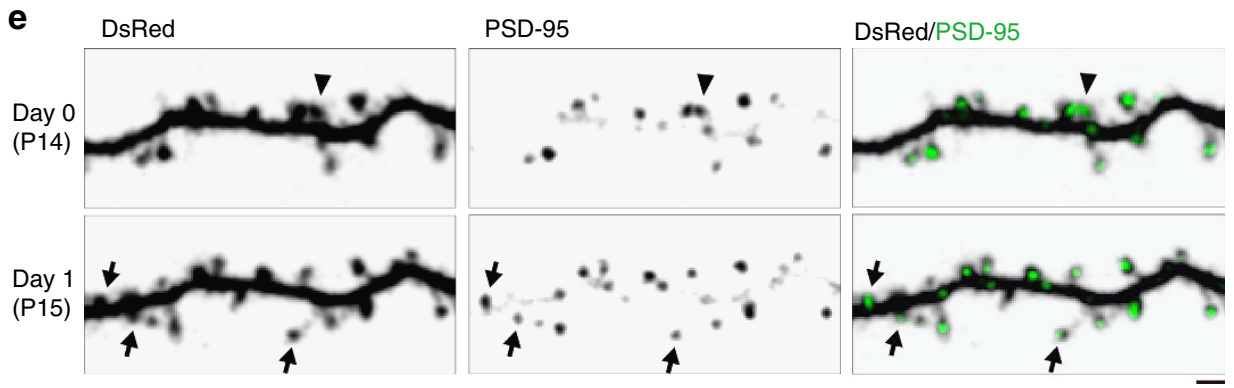

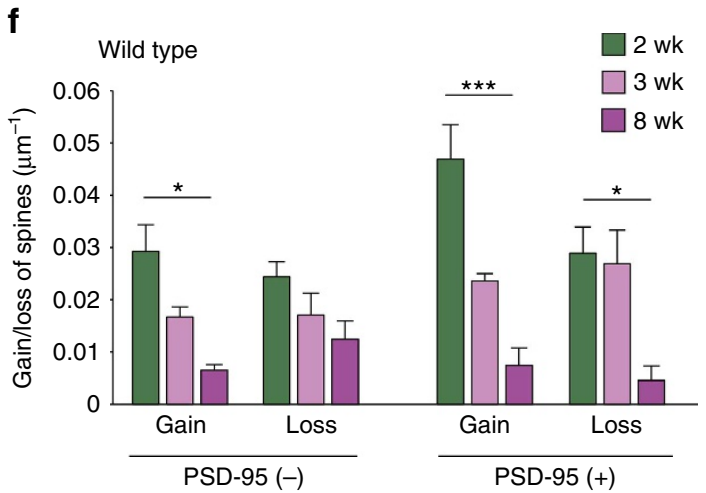

Figure 1 | Characterization of PSD-95-GFP-( + ) and $(-\mathbf{~}$ ) spines. (a) Postnatal development of dendritic spines and PSD-95 clusters in wild-type mice. Imaging of PSD-95-GFP (green) and DsRed2 (grey) revealed PSD-95-GFP- $(+$ ) (arrows) and PSD-95-GFP- $(-$ ) spines (arrowheads) in the SSC at different time points of postnatal development. (b) Postnatal increase of total spines and spines positive with PSD-95-GFP clusters in the SSC of wild-type mice. Error bars indicate s.e.m. of the spine densities classified as PSD-95-GFP- $(+)$ or PSD-95-GFP- $(-)$. (c) Turnover rates of total spines at different time points. (d) Distribution of spine volumes classified by the presence or absence of PSD-95-GFP clusters (PSD-95- + ) spines, $n=353$; PSD-95-( - ) spines, $n=43$ ). (e) In vivo imaging of PSD-95-GFP (grey in middle panels, green in right panels) and dendrites filled with DsRed2 (grey). Gain (arrows) and loss (arrowheads) of PSD-95-GFP- $(+)$ spines were detected. (f) Gain and loss of spines classified by the presence or absence of PSD-95-GFP clusters at three different time points during postnatal development in wild-type mice. Data are presented as mean \pm s.e.m. ${ }^{\star} P<0.05$,

${ }^{\star \star \star} P<0.005$, one-way analysis of variance, Tukey's-test. Refer to the Supplementary Table 1 for the number of animals in each group. Scale bars, $2 \mu$ m.

In vivo time-lapse imaging over 1 day at postnatal weeks 2,3 and 8 revealed important properties of PSD-95-GFP- $(+)$ spines (Fig. 1e,f). First, the dynamics of PSD-95-GFP- $(+)$ spines were developmentally downregulated. As the fractions of spines without PSD-95 clusters were small, the suppression of
PSD-95-GFP- $(+)$ spine dynamics underlay the downregulation of total spine turnover. Second, acquisition of PSD-95-GFP clusters within pre-existing PSD-95-GFP- $(-)$ spines cannot explain the appearance of PSD-95-GFP- $(+)$ spines. Within $24 \mathrm{~h}$, the number of newly formed PSD-95-GFP- $(+)$ spines was 
$2.36 \pm 0.14$ per $100 \mu \mathrm{m}$ of dendrites at postnatal week 3 (Fig. 1f). However, the transition events from PSD-95-GFP- $(-)$ to $(+)$ spines were less frequent $(0.73 \pm 0.08$ per $100 \mu \mathrm{m}$ of dendrites; 12 events per 655 spines, $n=4$ animals), indicating that a large fraction of PSD-95-GFP- $(+)$ spines were generated de novo within $24 \mathrm{~h}$. Taken together with the developmental profiles of PSD-95-GFP- $(+)$ spine increase (Fig. 1b), we concluded that a turnover of PSD-95-GFP- $(+)$ spines and its developmental regulation mainly contribute to the formation of stable neural circuits in the mature neocortex.

The second marker we used for spine classification is gephyrinGFP (Fig. 2a). Layer 2/3 pyramidal neurons receive excitatory afferents from multiple neuronal populations. A subset of spines receive inhibitory inputs from local interneurons, and these spines also form excitatory synaptic connections with TCAs ${ }^{37}$. Therefore, the identification of spines containing gephyrin-GFP may be useful in the grouping of heterogeneous spine populations. A majority of gephyrin-GFP- $(+)$ spines $(63 \%)$ were apposed to puncta immunoreactive with VGluT2, a reliable marker of presynaptic terminals of TCAs (Fig. 2b,c). In contrast, the association of gephyrin-GFP- $(+)$ spines with VGluT1, a marker of intracortical axon terminals, was at chance level $(31 \%$ versus $37 \%$ for image-shifted controls). Conversely, we confirmed the preferential apposition of gephyrin-GFP- $(-)$ spines to VGluT1-positive terminals (62\% of gephyrin-GFP-( - ) spines), while apposition of gephyrin-GFP- $(-)$ spines to VGluT2positive terminals was at chance level (29\% versus $32 \%$ for image-shifted controls) (Fig. 2b,c). Colocalization of gephyrinGFP- $(+)$ spines with VGluT2 immunoreactivity was comparable to that of spines positive with endogenous gephyrin clusters $(67 \%$ $(18 / 27)$ of spines identified by expression of DsRed), suggesting that gephyrin-GFP reported distribution of endogenous molecules. Gephyrin-GFP- $(+)$ spines were $20 \%$ of the total spines and larger than gephyrin-GFP- $(-)$ spines (Fig. 2d,e), further supporting their distinct properties. Our quantitative analysis of spine volume suggests little overlap between PSD-95GFP- $(-)$ and gephyrin-GFP- $(+)$ spines (Figs $1 d$ and 2e). Approximately $90 \%$ of spines with gephyrin immunoreactivity showed colocalization with PSD-95-GFP clusters (Supplementary Fig. 1b). We concluded that gephyrin-positive spines were a subset of PSD-95-positive spines.

We next examined the dynamics of gephyrin-GFP- $(+)$ and ( - ) spines by in vivo imaging of mice at postnatal week 3 . In vivo time-lapse imaging over 2 days at postnatal week 3 revealed distinct dynamic properties of gephyrin-GFP- $(+)$ and $(-)$ spines (Fig. 2f). Gephyrin-GFP- $(+)$ spines were extremely stable compared with gephyrin-GFP- $(-)$ spines (Fig. 2g). As the turnover rates of gephyrin-GFP- $(+)$ spines were lower than those of total spines at postnatal week 8 (Figs $1 \mathrm{f}$ and $2 \mathrm{~g}$ ), the differences in the extent of differentiation cannot explain the stability of the gephyrin-GFP- $(+)$ spines. From these observations, we concluded that the turnover of gephyrin-GFP- $(+)$ and $(-)$ spines reflected distinct dynamic properties of thalamocortical synapses and intracortical synapses onto the same postsynaptic dendrites of layer $2 / 3$ neurons.

Enhanced turnover of specific spine subtypes in patDp/+ mice. The in vivo visualization technique of spines marked by either excitatory or inhibitory postsynaptic scaffolds was applied to the detection of synaptic phenotypes in ASD mouse models. We first analysed a mouse model of non-syndromic ASD, in which the chromosomal region corresponding to human $15 \mathrm{q} 11-13$ is paternally duplicated (patDp/ + mice) ${ }^{24}$. There were no apparent abnormalities in the architecture of SSC (Supplementary Fig. 2), the density of spines (Fig. 3a,b) and fluorescence intensities of PSD-95-GFP and gephyrin-GFP puncta (Supplementary Fig. 3a,c) in patDp/ + mice. We performed in vivo imaging and measured the turnover of spines marked by PSD-95-GFP in the SSC of wild-type and patDp/ + mice over 2 days at postnatal week 3 . We found that the turnover of PSD-95-GFP- $(+)$ spines was significantly enhanced (Fig. 3c), and the gain and loss of PSD-95-GFP- $(+)$ spines increased in patDp/ + mice (Fig. 3d). However, the dynamics of PSD-95GFP- $(-)$ spines were comparable between patDp/ + and wild-type mice (Fig. 3c,d). These results indicated that genetic modifications mimicking the duplication of human chromosome 15q11-13 preferentially affected the remodelling of PSD-95-GFP$(+)$ spines, which contributed to the establishment of stable neural circuits in the SSC.

We next performed in vivo imaging of spines marked by gephyrin-GFP in wild-type and patDp/ + mice (Fig. 3e). No significant differences were present in the fraction of spines positive with gephyrin-GFP between $\mathrm{patDp} /+$ and wild-type mice (Fig. 3f). Our in vivo imaging over 2 days revealed that the dynamics of gephyrin-GFP- $(-)$ spines were significantly enhanced in $\mathrm{patDp} /+$ mice, whereas the stability of gephyrinGFP- $(+)$ spines were comparable between patDp/ + and wildtype mice (Fig. 3g,h). This indicates that genetic modifications mimicking the duplication of human chromosome 15q11-13 specifically affected the remodelling of gephyrin-GFP-( - ) spines, which mainly receive inputs from other cortical neurons. As patDp/ + mice showed enhanced turnover in PSD-95-GFP- $(+)$ and gephyrin-GFP- $(-)$ spines, we concluded that a specific subset of spines differentiating excitatory synaptic contacts with intracortical afferents showed enhanced turnover in patDp/ + mice.

Spine dynamics at different cortical areas and ages. We detected an enhanced turnover of PSD-95-GFP- $(+)$ spines as a major synaptic phenotype in the SSC of patDp/ + mice. We aimed to determine whether the synaptic phenotypes observed in the SSC at postnatal week 3 could be generalized to different cortical areas and developmental stages. Several lines of evidence indicated that the rodent anterior frontal cortex (AFC) may be defective in ASD mouse models. First, studies in patients with ASD indicated dysfunction and early postnatal overgrowth in the frontal cortex ${ }^{38}$. Second, pyramidal neurons in the AFC showed hyperconnectivity in an ASD mouse model ${ }^{39}$. Third, from PND 2 to 21 , the $\mathrm{AFC}$ receives a large number of long-distance intracortical projections ${ }^{40}$, which are considered to be less developed in patients with $\mathrm{ASD}^{38}$. The AFC imaged in this study was anterior to the primary motor cortex ${ }^{41}$ and distinct from the rodent prefrontal cortex ${ }^{42}$ (Supplementary Fig. 4). The density of spines in the AFC was not different between wild-type and patDp/ + mice (Fig. 4a,b). We found that the dynamics of PSD-95-GFP- $(+)$ spines was enhanced in the AFC of patDp/ + mice (Fig. 4c) and both the gain and loss of PSD-95-GFP- $(+)$ spines increased (Fig. 4d). Upregulation of spine turnover was comparable between the AFC and SSC, indicating the presence of autism-related synaptic phenotypes in multiple cortical areas (compare Figs 3c with 4c).

We next performed in vivo imaging of $\mathrm{patDp} /+$ mice over 1 day at postnatal week 2 (Fig. 4e,f). We found that the gain and loss of PSD-95-GFP- $(+)$ spines were also upregulated in patDp $/+$ mice at postnatal week 2 (Fig. $4 \mathrm{~g}, \mathrm{~h}$ ). In wild-type mice, the gain of PSD-95-GFP- $(+)$ spines exceeded their loss at this time point (Fig. 4h), and this relationship was reversed at postnatal week 3 (Fig. 3d). This shift in the balance between spine gain and loss was preserved in patDp/ + mice (Figs $3 \mathrm{~d}$ and $4 \mathrm{~h}$ ). The rates of gain and loss of PSD-95-GFP- $(-)$ spines were also 
a

P23

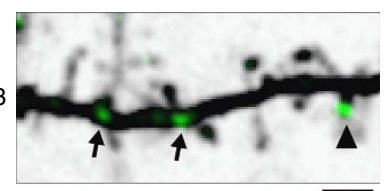

b

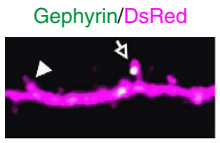

VGluT1/DsRed

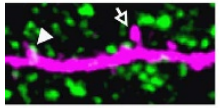

VGluT1/gephyrin/DsRed

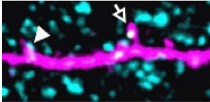

C

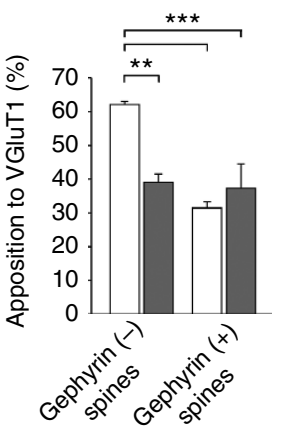

Gephyrin/DsRed

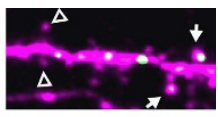

VGluT2/DsRed

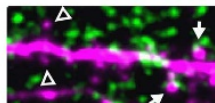

VGluT2/gephyrin/DsRed

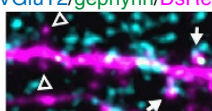

$\Delta, x^{2}, x^{2}$
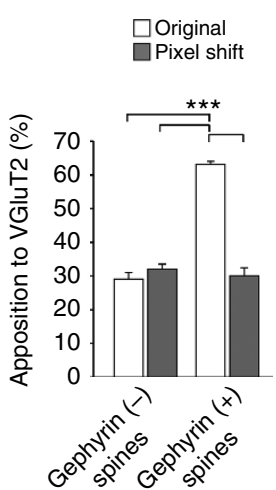

d

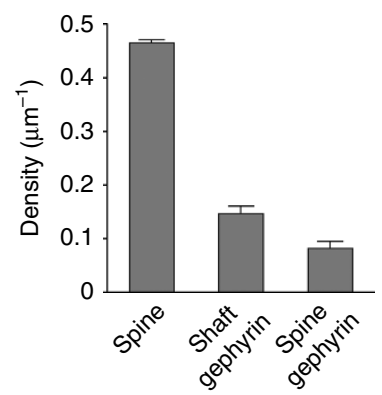

e

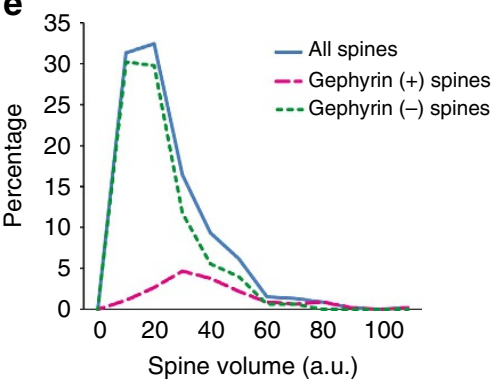

f

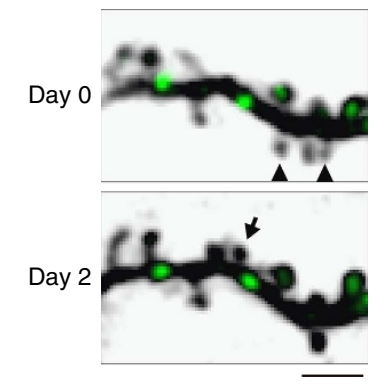

g

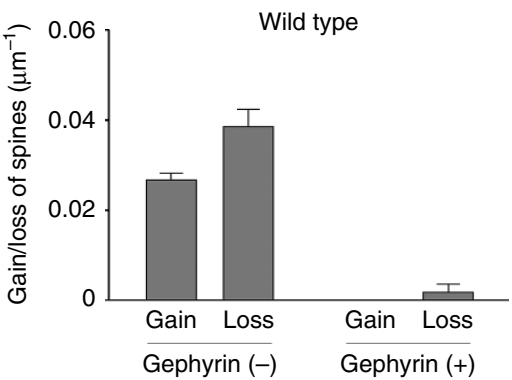

Figure 2 | Characterization of gephyrin-GFP-( + ) and (-) spines. (a) Imaging of gephyrin-GFP (green) and DsRed2 (grey) revealed that gephyrin-GFP localized on dendritic shafts (arrows) and spines (arrowhead) in layer 2/3 pyramidal neurons of wild-type mice. (b) VGluT1 or VGluT2 (middle, green; lower, blue) immunocytochemistry of the SSC containing neurons expressing DsRed2 (magenta) and gephyrin-GFP (green) by in utero electroporation. Open arrow: Gephyrin-GFP- $(+)$ spine without a contact with VGluT1 puncta. Solid arrows: Gephyrin-GFP- $(+)$ spines juxtaposed to VGluT2 puncta. Open arrowheads: Gephyrin-GFP- $(-)$ spines without contacts with VGluT2 puncta. Solid arrowhead: Gephyrin-GFP-( - ) spine juxtaposed to a VGluT1 punctum. (c) Percentage of gephyrin-GFP- $(+)$ or $(-)$ spines in close apposition to VGluT1 and VGluT2 puncta. To estimate the apposition of fluorescent puncta at chance level, the GFP channel was shifted by 50 pixels (pixel shift) and the percentage of apposition was calculated (VGluT1, $n=3$ animals; VGluT2, $n=3$ animals). (d) Densities of dendritic spines, shaft gephyrin and spine gephyrin clusters per unit length of dendrites in the SSC of wild-type mice. (e) Distribution of spine volumes classified by the presence or absence of gephyrin-GFP clusters (gephyrin-GFP- $(+)$ spines, $n=80$; gephyrin-GFP- $(-)$ spines, $n=337$ ). (f) In vivo imaging of gephyrin-GFP (green) and dendrites filled with DsRed2 (grey). Gain (arrow) and loss (arrowheads) of gephyrin-GFP- $(-)$ spines were detected. (g) Spines were classified by the presence or absence of gephyrin-GFP, and the rates of gain and loss of spines per unit length of dendrites were measured. Data are presented as mean \pm s.e.m. ${ }^{\star \star} P<0.01$, ${ }^{\star \star \star} P<0.005$, Student's $t$-test. Refer to the Supplementary Table 1 for the number of animals in each group. Scale bars, $3 \mu \mathrm{m}$.

enhanced at postnatal week 2 (Fig. $4 \mathrm{~h}$ ), suggesting the presence of a developmental stage-specific phenotype in patDp/ + mice. In summary, enhanced turnover of PSD-95-GFP- $(+)$ spines is likely to be a common phenotype across different cortical areas and developmental stages.
Shared spine phenotypes in patDp/ + and NLG R451C mice. If multiple mouse models of non-syndromic ASDs share similar defects in synapse remodelling, this information in similarity should be useful in defining the core characteristics of ASDs. To test whether the enhanced turnover of synapses was present in 
a

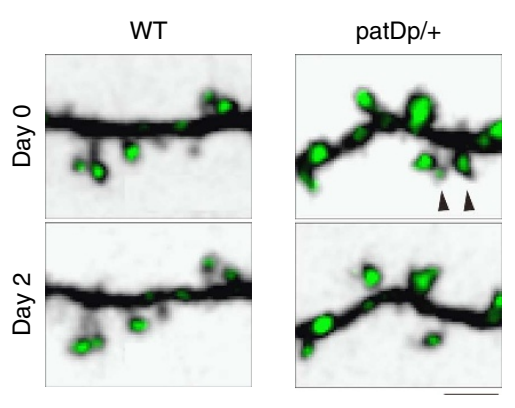

C

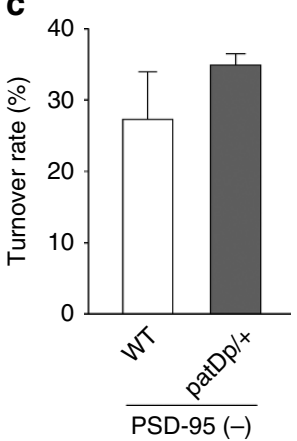

e
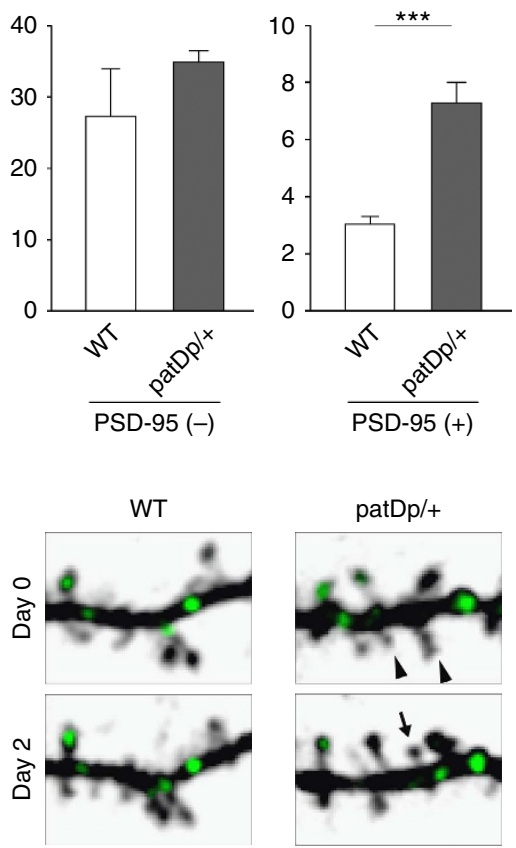

patDp/+

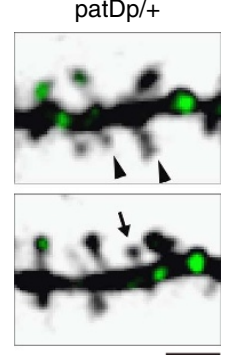

g

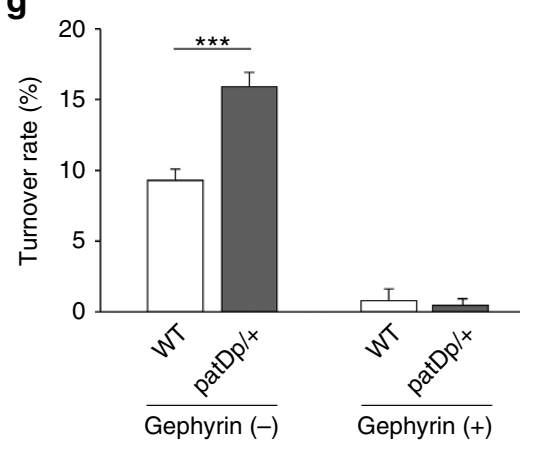

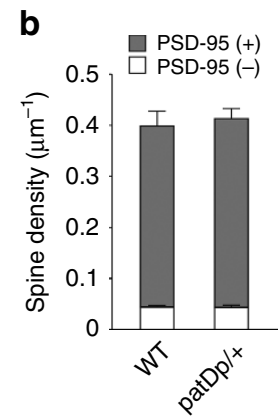

d

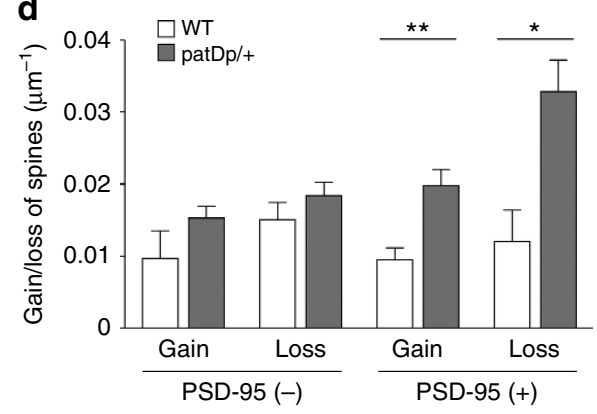

f

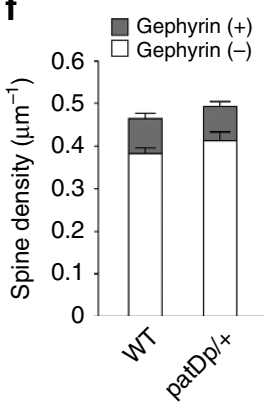

h

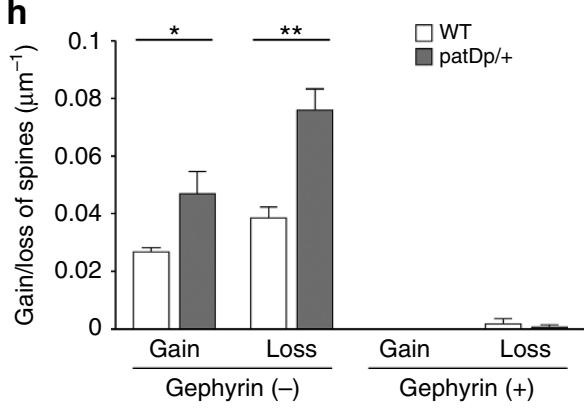

Figure 3 | Upregulation of synapse turnover in patDp/ + mice. (a) In vivo imaging of PSD-95-GFP (green) and dendrites filled with DsRed2 (grey) in the SSC of patDp/ + mice. Loss of PSD-95-GFP- $(+)$ spines was detected (arrowheads). (b) Spine density with or without PSD-95-GFP clusters in the SSC of wild-type and patDp/ + mice. Error bars indicate s.e.m. of the spine densities classified as PSD-95-GFP- $(+)$ or PSD-95-GFP- $(-)$.

(c) Turnover of PSD-95-GFP- $(+)$ and ( - ) spines over 2 days in the SSC of wild-type and patDp/ + mice. (d) Gain and loss of PSD-95-GFP- $(+)$ and ( - ) spines per unit length of dendrites over 2 days in the SSC of wild-type and patDp/ + mice. (e) In vivo imaging of gephyrin-GFP (green) and dendrites filled with DsRed2 (grey). Gain (arrow) and loss (arrowheads) of spines were detected. (f) Spine density with or without gephyrin-GFP clusters in the SSC of wild-type and patDp/ + mice. Error bars indicate the s.e.m. of the spine densities classified as gephyrin-GFP- $(+)$ or gephyrin-GFP- $(-)$.

(g) Turnover of gephyrin-GFP- $(+)$ and $(-)$ spines over 2 days in the SSC of wild-type and patDp/ + mice. (h) Gain and loss of gephyrin-GFP- $(+)$ and $(-)$ spines per unit length of dendrites over 2 days in the SSC of wild-type and patDp/ + mice. Data are presented as mean \pm s.e.m. ${ }^{\star} P<0.05$,

${ }^{\star \star} P<0.01,{ }^{\star \star \star} P<0.005$, Student's $t$-test. Refer to the Supplementary Table 2 for the number of animals in each group. Scale bars, $3 \mu \mathrm{m}$.

other mouse models, we next analysed NLG R451C mice $^{15}$, which were engineered to have a rare missense mutation of NLGN-3 found in non-syndromic ASDs. We first evaluated the spine density and dynamics of PSD-95-GFP- $(+)$ spines in the AFC at postnatal week 3 (Fig. 5a). Although the density of spines (Fig. 5b) and fluorescence intensity of PSD-95-GFP puncta (Supplementary Fig. 3b) were not altered in the AFC of NLG $\mathrm{R} 451 \mathrm{C}$ mice, in vivo imaging of NLG R451C mice showed 
a

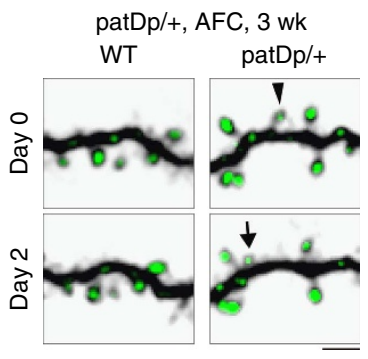

C
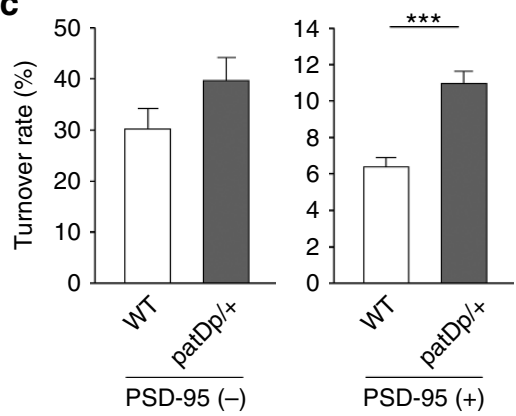

e

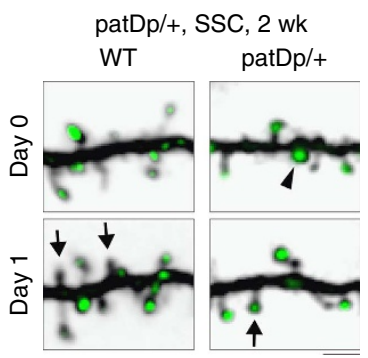

g
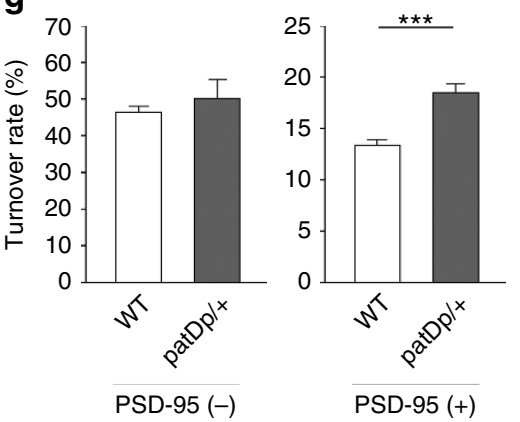
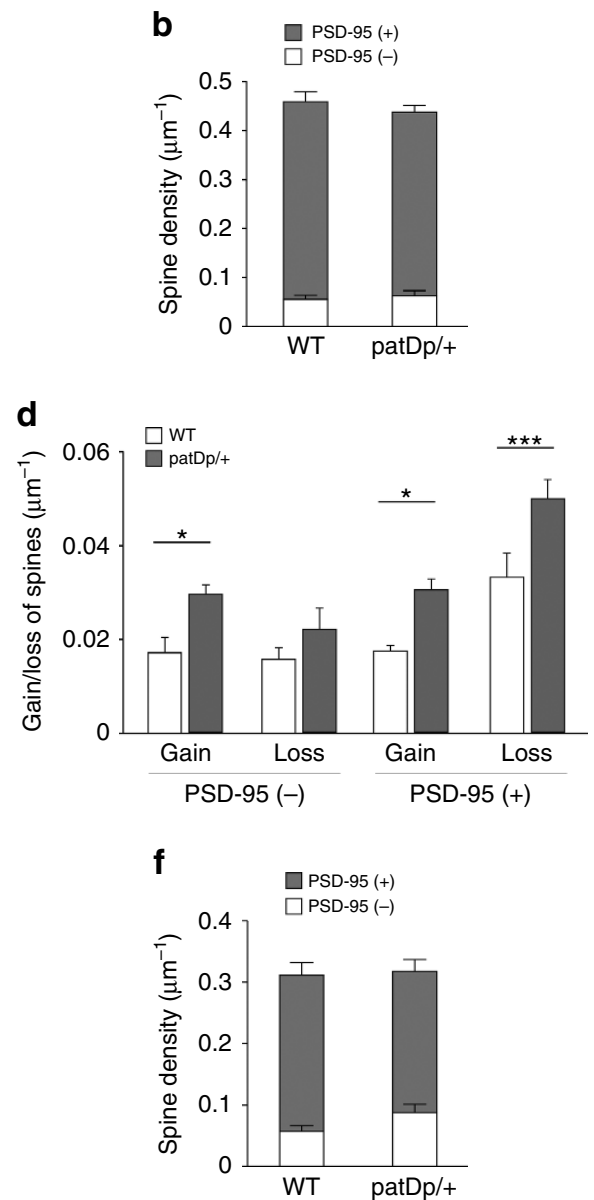

h

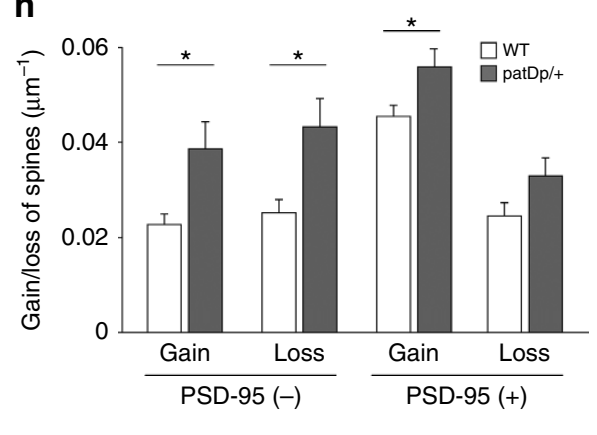

Figure 4 | patDp/ + mice show enhanced spine dynamics in the AFC at postnatal week 3 and in the SSC at postnatal week 2. (a) In vivo time-lapse imaging of PSD-95-GFP (green) and dendrites filled with DsRed2 (grey) over 2 days in the AFC of wild-type and patDp/ + mice. Gain (arrow) and loss (arrowhead) of PSD-95-GFP- $(+)$ spines were detected. (b) Spine density with or without PSD-95-GFP clusters in the AFC of wild-type and patDp/ + mice. Error bars indicate the s.e.m. of the spine densities classified as PSD-95-GFP- $(+)$ or PSD-95-GFP- $(-)$. (c) Turnover of PSD-95-GFP- $(+)$ and $(-)$ spines over 2 days in the AFC of wild-type and patDp/ + mice at postnatal week 3. (d) Gain and loss of PSD-95-GFP- $(+)$ and $(-)$ spines per unit length of dendrites in the AFC of wild-type and patDp/ + mice at postnatal week 3. (e) In vivo time-lapse imaging of PSD-95-GFP (green) and dendrites filled with DsRed2 (grey) in the SSC of wild-type and patDp/ + mice at postnatal week 2. Gain (arrows) and loss (arrowhead) of spines were detected. (f) Spine density with or without PSD-95-GFP clusters in the SSC of wild-type and patDp/ + mice at postnatal week 2. Error bars indicate the s.e.m. of the spine densities classified as PSD-95-GFP- $(+)$ or PSD-95-GFP- $(-)$. (g) Turnover of PSD-95-GFP- $(+)$ and $(-)$ spines over 1 day in the SSC of wild-type and patDp/ + mice at postnatal week 2. (h) Gain and loss of PSD-95-GFP- $(+)$ and $(-)$ spines per unit length of dendrites in the SSC of wild-type and patDp/ + mice at postnatal week 2. Data are presented as mean \pm s.e.m. ${ }^{\star} P<0.05$, ${ }^{\star \star \star} P<0.005$, Student's $t$-test. Refer to the Supplementary Table 2 for the number of animals in each group. Scale bars, $3 \mu \mathrm{m}$.

enhanced dynamics of PSD-95-GFP- $(+)$ spines (Fig. 5c,d). Upregulation of spine turnover was comparable between NLG $\mathrm{R} 451 \mathrm{C}$ and $\mathrm{patDp} /+$ mice, indicating the presence of the autism-related common synaptic phenotype in the AFC of multiple mouse models. The gain and loss of PSD-95-GFP- $(+)$ spines significantly increased in the AFC of NLG R451C mice (Fig. 5d).

To further evaluate the similarities of synaptic dynamics in multiple ASD mouse models, we next tested whether spines classified by the presence of gephyrin-GFP clusters in 
a

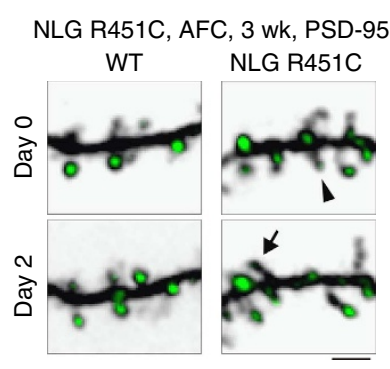

C

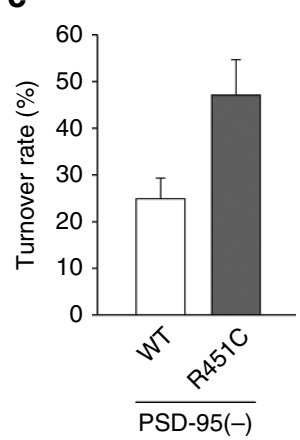

e

NLG R451C, SSC, 3 wk, gephyrin WT NLG R451C

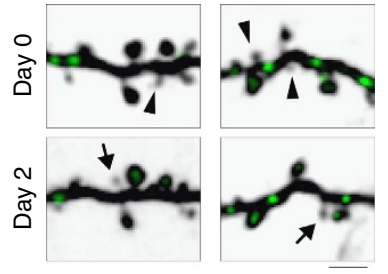

g

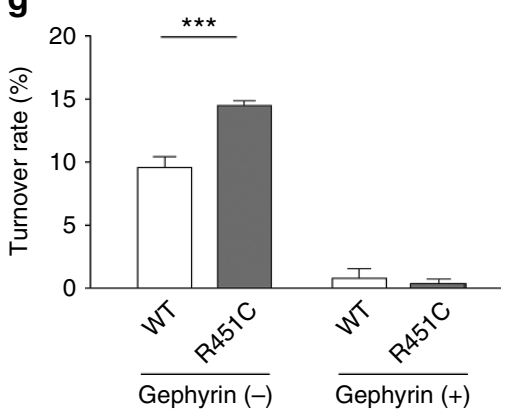

b

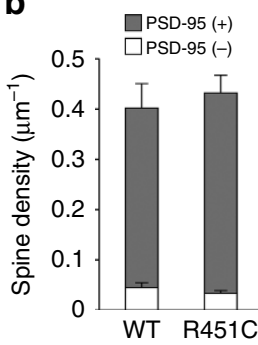

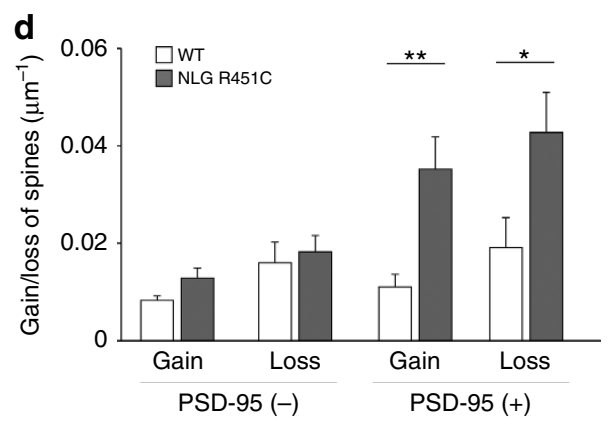

f
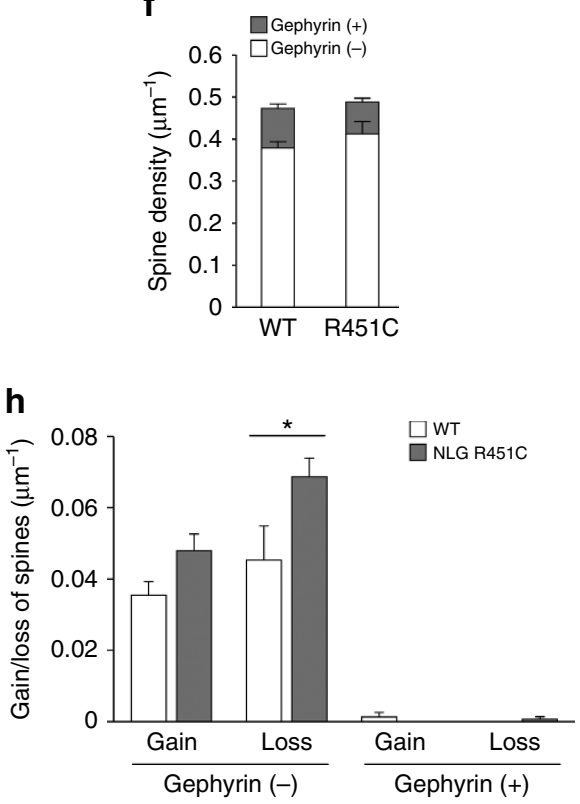

Figure 5 | Upregulation of spine turnover in NLG R451C mice. (a) In vivo time-lapse imaging of PSD-95-GFP (green) and dendrites filled with DsRed2 (grey) over 2 days in the AFC of wild-type and NLG R451C mice. Gain (arrow) and loss (arrowhead) of PSD-95-GFP- $(+$ ) spines was detected. (b) Spine density with or without PSD-95-GFP clusters in the AFC of wild-type and NLG R451C mice. Error bars indicate the s.e.m. of the spine densities classified as PSD-95-GFP- $(+)$ or PSD-95-GFP- $(-)$. (c) Turnover of PSD-95-GFP- $(+)$ and $(-)$ spines over 2 days in the AFC of wild-type and NLG R451C mice. (d) Gain and loss of PSD-95-GFP- $(+)$ and $(-)$ spines per unit length of dendrites over 2 days in the AFC of wild-type and NLG R451C mice. (e) In vivo time-lapse imaging of gephyrin-GFP (green) and dendrites filled with DsRed2 (grey) over 2 days in the SSC of wild-type and NLG R451C mice. Gain (arrows) and loss (arrowheads) of gephyrin-GFP- $(-)$ spines were detected. (f) Spine density with or without gephyrin-GFP clusters in the SSC of wild-type and NLG R451C mice. Error bars indicate the s.e.m. of the spine densities classified as gephyrin-GFP- $(+)$ or gephyrin-GFP- $(-)$. (g) Turnover of gephyrin-GFP- $(+)$ and $(-)$ spines over 2 days in the SSC of wild-type and NLG R451C mice. (h) Gain and loss of gephyrin-GFP- $(+)$ and $(-)$ spines per unit length of dendrites over 2 days in the SSC of wild-type and NLG R451C mice. Data are presented as mean \pm s.e.m. ${ }^{\star} P<0.05$, ${ }^{\star \star} P<0.01$,

${ }^{\star \star \star} P<0.005$, Student's $t$-test. Scale bars, $3 \mu \mathrm{m}$. Refer to the Supplementary Table 3 for the number of animals in each group.

the SSC showed similar defects in NLG R451C mice (Fig. 5e). The densities of gephyrin-GFP- $(+)$ and $(-)$ spines (Fig. 5f), and fluorescence intensity of gephyrin-GFP puncta (Supplementary Fig. 3d) in the SSC were comparable between NLG R451C and wild-type mice. Similar to patDp/ + mice, in vivo imaging showed that the turnover of gephyrin-GFP- $(-)$ spines was enhanced in NLG R451C mice, whereas gephyrinGFP- $(+)$ spines were highly stable and their dynamics were comparable between wild-type and NLG R451C mice (Fig. 5g,h). 
Our in vivo imaging experiments using two mouse models of non-syndromic ASDs revealed remarkably similar alterations in the dynamics of specific spine subsets. We further extended in vivo imaging analyses of PSD-95-GFP- $(+)$ spines to the third ASD mouse model, BTBR mice ${ }^{43}$. Dynamics of PSD-95-GFP$(+)$ spines in the AFC of BTBR mice at postnatal week 3 was once more upregulated to a similar extent (Supplementary Fig. 5). We concluded that the enhanced turnover of PSD-95-GFP- $(+)$ spines that participate in the intracortical connectivity underlay autism-related deficits in the cortex of multiple ASD mouse models.

Dynamics of inhibitory synapses in patDp/ + mice. To investigate whether the turnover of inhibitory synapses per se is altered in ASD mouse models, we measured the number of newly added and lost gephyrin clusters in patDp/ + and NLG R451C mice (Fig. 6a,b). One-third of gephyrin-GFP clusters were localized within spines and the remaining two-thirds were on dendritic shafts (Fig. 2d). The densities of shaft gephyrin clusters in patDp/ + and NLG R451C mice were comparable with wild-type mice (Fig. 6c,f). In vivo imaging of the SSC over 2 days at postnatal week 3 revealed enhanced dynamics of shaft gephyrin clusters in patDp/ + mice (Fig. 6d), whereas NLG R451C mice showed dynamics of shaft gephyrin clusters comparable with wild-type mice (Fig. $6 \mathrm{~g}$ ). The turnover of spine gephyrin clusters was not altered in both ASD mouse models (Fig. 6e,h). The observed changes in the shaft gephyrin clusters may be related to the specific impairment of neuronal functions present in patDp/ + mice.

Relative distances between spines and inhibitory synapses may influence the stability of spines. Shaft gephyrin clusters were more stable than spine gephyrin clusters, and the fluorescence intensities of shaft clusters were higher than those of the spine clusters (Supplementary Fig. 6). Therefore, we focused on shaft gephyrin clusters and grouped spines into those within $4 \mu \mathrm{m}$ and those more than $4 \mu \mathrm{m}$ away from shaft gephyrin clusters. Although spine volume was not different between two groups (Supplementary Fig. 7e), spines closer to shaft gephyrin clusters were less stable (Supplementary Fig. 7a-d). This effect was not due to differential distribution of gephyrin-positive stable spines between two groups (Supplementary Fig. 7f). Furthermore, this effect of nearby gephyrin clusters on spine stability was abolished in both patDp/ + and NLG R451C mice. These results indicated that both the dynamics of inhibitory synapses and the influence of inhibitory synapses on nearby spines can be affected by genetic mutations related to ASDs.

Experience-dependent regulation of spine dynamics. Alterations of spine dynamics in ASD mouse models may influence activity-dependent regulation of neural network development in the neocortex. Sensory inputs of the barrel cortex can be modulated by trimming whiskers in a chess-board pattern ${ }^{44}$. In vivo imaging of gephyrin-GFP- $(+)$ and $(-)$ spines in the barrel cortex over 2 days at postnatal week 3 , combined with chess-board whisker trimming, revealed increase in gain of spines receiving inputs from intracortical neurons in wild-type mice ${ }^{44,45}$ (Fig. 7a,b). Gephyrin-GFP- $(+)$ spines were not affected by whisker trimming, indicating that intracortical circuit is selectively influenced by sensory modulation. In patDp/+ mice, chess-board whisker trimming did not affect gain of gephyrin-GFP- $(-)$ spines (Fig. 7b). Similar to patDp/ + mice, chess-board trimming of whiskers did not affect gain of gephyrinGFP- $(-)$ spines in NLG R451C mice (Fig. 7c). Gephyrin-GFP$(+)$ spines were highly stable in all experimental conditions (Fig. 7b,c).
Lack of experience-dependent regulation of spine formation in ASD mouse models may be associated with altered neuronal activity driven by sensory inputs. To test this, we controlled sensory inputs to different rows in the barrel cortex by trimming all whiskers, except row $\mathrm{C}$, and exposure to an enriched environment for $1 \mathrm{~h}$ (ref. 46). Subsequent immunohistochemistry with c-fos antibody revealed increases in the number of c-fospositive cells in the cortical columns corresponding to row $\mathrm{C}$ (Fig. $7 d$ ). The density of c-fos-positive cells in the layer $2 / 3$ was reduced in both patDp/ + mice and NLG R451C mice. Responses of layer 4 neurons to whisker stimulation were not affected in ASD mouse models (Fig. 7e,f). These results support the idea that patDp/ + and NLG R451C mice share the common defects in neural circuit development responsible for sensory processing within the cortex.

\section{Discussion}

During development, neural circuits are established with extensive remodelling of synapses. Our imaging study using multiple ASD mouse models suggests that an enhanced turnover of excitatory synapses is a common phenotype of non-syndromic ASD mouse models. Detailed analyses of spine subtypes using an inhibitory postsynaptic marker, gephyrin, revealed that a subset of spines associated with afferents from cortical neurons exhibited enhanced turnover. These results suggest that abnormal dynamics of a specific population of synapses during development underlie cortical dysfunctions in ASDs.

We found an enhanced turnover of spines in ASD mouse models with distinct genetic backgrounds. In patDp/ + mice, genes such as Ndn, Snrpn, Gabra5, Gabr $\beta 3$ and Gabr $\gamma 3$ are upregulated $^{24}$. Altered RNA editing and calcium influx through 5-HT2c receptors by abnormal small nucleolar RNA expression were proposed to be a candidate mechanism related to autism ${ }^{24}$. NLG R451C mice display an enhancement of mIPSC frequency and increased expression of inhibitory synapse-related proteins ${ }^{15}$. As neuroligin-3 is localized to both excitatory and inhibitory synapses ${ }^{47}$, the enhancement of spine dynamics may result from abnormal function of neuroligin-3 in excitatory synapses. BTBR mice, the inbred strain identified by behavioural screening to mimic the core behavioural deficits of ASD, also showed a similar spine phenotype. In addition to these three ASD mouse models that were analysed in this study, the Fmrl knockout (KO) mice, a fragile $\mathrm{X}$ syndrome and syndromic autistic mouse model, displayed enhanced spine turnover ${ }^{45,48}$, further supporting the idea that an increase in synapse turnover is a common phenotype across diverse ASD mouse models.

How do diverse genetic mutations in these ASD mouse models lead to a highly similar phenotype in the turnover of excitatory synapses? Synapse turnover is regulated by both activitydependent and -independent pathways ${ }^{32,34,49}$. Synapses can still be generated without activity-dependent processes, but the process of synapse stabilization may be selectively affected ${ }^{50}$. If diverse genetic mutations in these mouse models converge to impairment of activity-dependent stabilization, mice models may show similar enhancement in synapse turnover. An obvious candidate pathway is $\mathrm{N}$-methyl-D-aspartate (NMDA) receptordependent signalling ${ }^{51}$, and previous studies indicated defects in NMDA receptor-dependent synaptic plasticity in ASD mouse models, including NLG R451C mice ${ }^{52}$.

In this study, we found that enhanced spine turnover was restricted to gephyrin-GFP- $(-)$ spines receiving intracortical projections in the SSC at postnatal week 3. The enhancement of gephyrin-GFP- $(-)$ spines was observed in both patDp/ + and NLG R451C mice. Thus, this selective impairment may be a common property of ASD mouse models. Gephyrin-GFP- $(+)$ and $(-)$ spines were present within the same dendritic segments, 
a

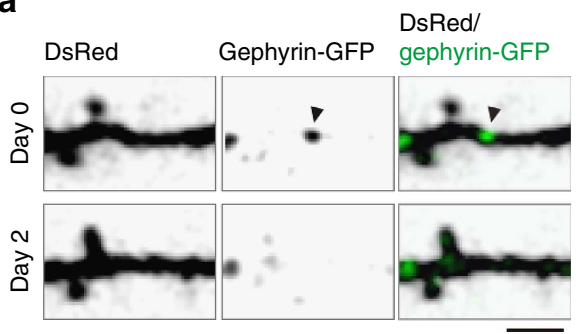

b

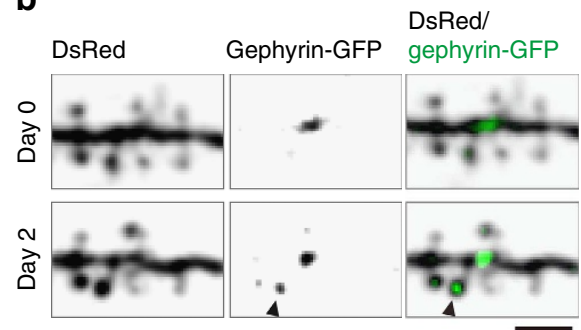

C

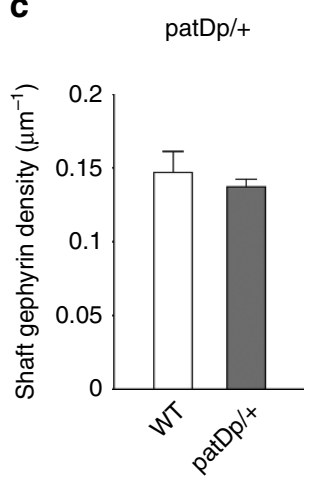

f

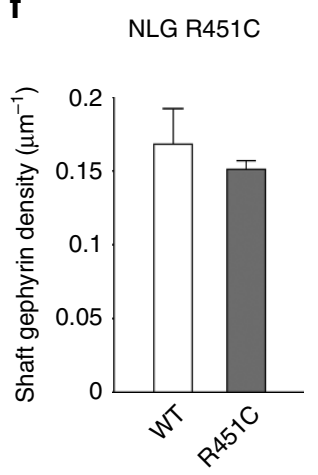

d

d patDp/+

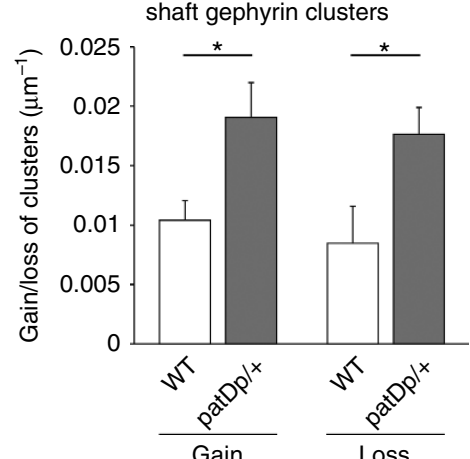

g

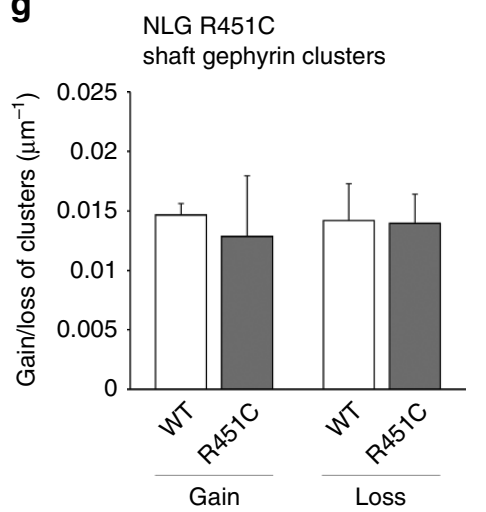

e

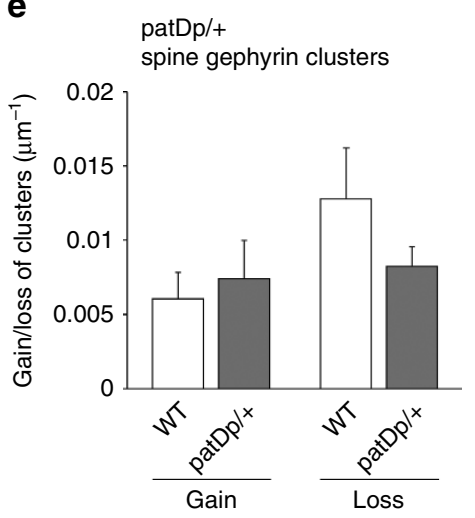

h

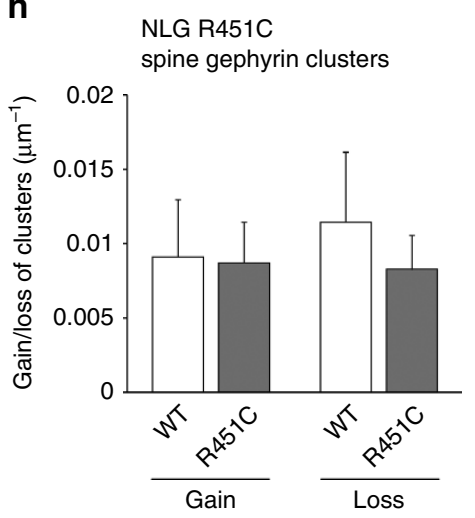

Figure 6 | Dynamics of shaft and spine gephyrin clusters in patDp/ + mice and NLG R451C mice. (a) An example of dynamic gephyrin-GFP clusters (grey in middle panels, green in right panels) on the dendritic shaft. Loss of a gephyhrin-GFP cluster was detected (arrowhead). (b) An example of dynamic gephyrin-GFP clusters (grey in middle panels, green in right panels) on dendritic spines. Gain of a gephyhrin-GFP cluster was detected (arrowhead). (c) Density of gephyrin clusters on dendritic shafts in the SSC of wild-type and patDp/ + mice. (d) Gain and loss of gephyrin clusters on dendritic shafts in the SSC of wild-type and patDp/ + mice. (e) Gain and loss of gephyrin clusters on dendritic spines in the SSC of wild-type and patDp/ + mice. (f) Density of gephyrin clusters on dendritic shafts in the SSC of wild-type and NLG R451C mice. (g) Gain and loss of gephyrin clusters on dendritic shafts in the SSC of wild-type and NLG R451C mice. (h) Gain and loss of gephyrin clusters on dendritic spines in the SSC of wild-type and NLG R451C mice. Data are presented as mean \pm s.e.m. ${ }^{\star} P<0.05$, Student's $t$-test. Scale bars, $3 \mu \mathrm{m}$. Refer to the Supplementary Tables 2 and 3 for the number of animals in each group.

but their dynamic properties were distinct. Large gephyrin-GFP$(+)$ spines were highly stable and unaffected by ASD-related gene mutations, while smaller gephyrin-GFP- $(-)$ spines were more dynamic and vulnerable to genetic manipulations mimicking ASDs. If the spine phenotypes identified in ASD mouse models were due to nonspecific deteriorative effects on postsynaptic pyramidal neurons, both types of spines should have been affected. Selective alterations in gephyrin-GFP- $(-)$ spines suggest important roles of presynaptic partners in the expression of ASD-related phenotypes. In addition, we found less significant effects of ASD-related genetic mutations on dynamics of PSD-95-GFP- $(-)$ spines. Spines without PSDs are likely to be immature spines without synaptic contacts. In ASD mouse models, an initial phase of spine morphogenesis may be preserved, but subsequent maturation processes, which are promoted by NMDA receptor-dependent signalling, may be affected, resulting in selective impairment of PSD-95-GFP- $(+)$ spines.

In wild-type mice, there was a large difference in stability of gephyrin-GFP- $(+)$ and $(-)$ spines, which receive distinct synaptic inputs from either VGluT2-positive thalamic or VGluT1-positive intracortical afferents. Differential spine dynamics may be explained by the different onset and closure of experience-dependent plasticity in two types of synaptic connections. Previous developmental studies of layer 4-layer 2/3 synapses indicated that synaptic plasticity begins and is most robust on PND12 - 14 but is present even in adulthood ${ }^{25,53}$. In the case of synapses between TCAs and layer 4 neurons, synaptic plasticity has been reported to occur only up to PND 7 (ref. 54). If synapses between TCAs and layer $2 / 3$ neurons have a time 
a

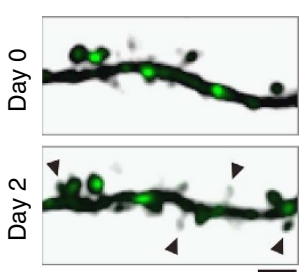

d

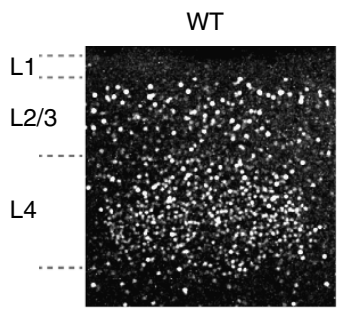

e b

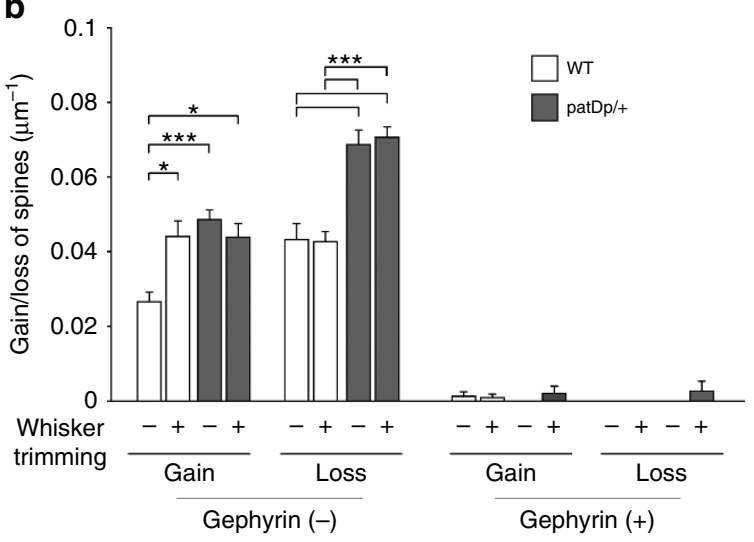

C

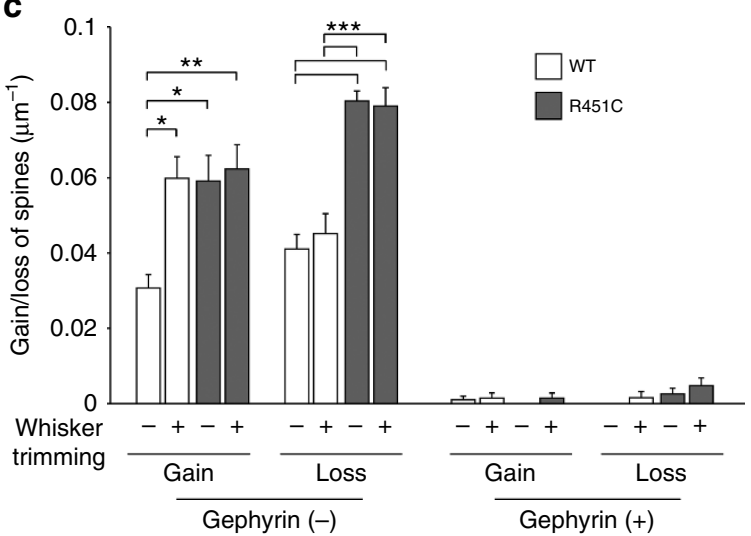

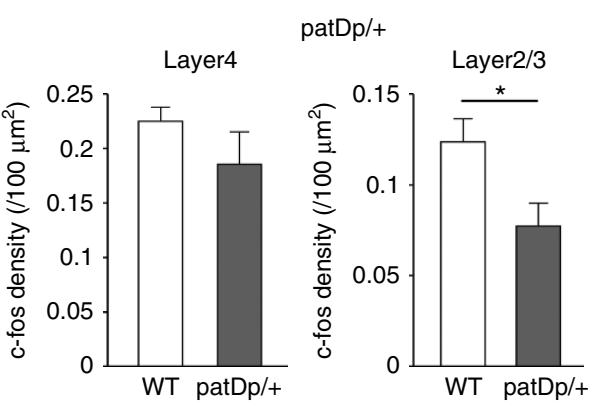

f

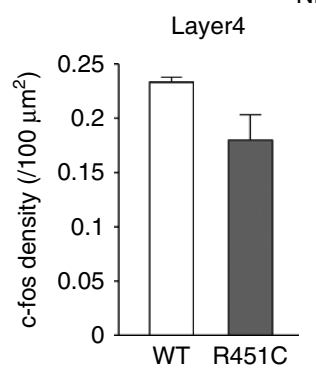

NLG R451C

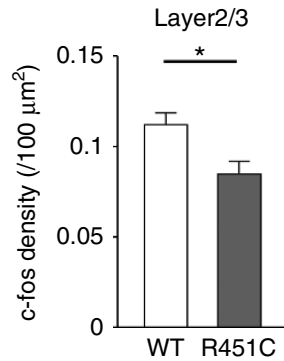

Figure 7 | Deficits of experience-dependent spine remodelling in ASD mouse models. (a) In vivo imaging of gephyrin-GFP (green) and dendrites filled with DsRed2 (grey). Chess-board trimming of whiskers promoted formation of new spines (arrowheads) in wild-type mice. (b) Gain and loss of gephyrinGFP- $(+)$ and $(-)$ spines per unit length of dendrites in the barrel cortex of wild-type and patDp/ + mice with or without chess-board trimming of whiskers. (c) Gain and loss of gephyrin-GFP- $(+)$ and $(-)$ spines per unit length of dendrites in the barrel cortex of wild-type and NLG R451C mice with or without chess-board trimming of whiskers. (d) Immunostaining of c-fos in the barrel cortex of wild-type and patDp/ + mice after whisker stimulation. (e) The density of c-fos immunoreactive cells in layer 4 (left) and layer $2 / 3$ (right) of wild-type and patDp/ + mice (WT, $n=4$ animals; patDp/ + , $n=4$ animals). (f) The density of c-fos immunoreactive cells in layer 4 (left) and layer 2/3 (right) of wild-type and NLG R451C mice (WT, $n=4$ animals; NLG R451C, $n=6$ animals). Data are presented as mean \pm s.e.m. ${ }^{\star} P<0.05$, ${ }^{\star \star} P<0.01,{ }^{\star \star \star} P<0.005$, Student's $t$-test. Scale bars, $3 \mu \mathrm{m}$ for $\mathbf{a} ; 100 \mu \mathrm{m}$ for $\mathbf{c}$. Refer to the Supplementary Table 4 for the number of animals in each group.

window of plasticity similar to those of layer 4 neurons, the differential dynamics between gephyrin-GFP- $(-)$ and gephyrinGFP- $(+)$ spines may be explained by the selective early closure of plasticity in TCAs-layer $2 / 3$ synapses. One can also argue that TCAs-layer $2 / 3$ synapses may have distinct properties. Layer 4 stellate cells and layer $2 / 3$ pyramidal cells are projected from different regions of the thalamus (ventral posteromedial nucleus to layer 4 neurons and medial posterior nucleus to layer $2 / 3$ neurons) ${ }^{55}$, and these projections transmit signals that have originated from distinct peripheral regions (lemniscal inputs through the ventral posteromedial nucleus and paralemniscal inputs through the medial posterior nucleus $)^{56}$. It is possible that TCAs-layer 2/3 synapses, which transmit global and multisensory information to the SSC, are less influenced by activity-dependent mechanisms of synapse remodelling. This may explain why
TCAs-layer $2 / 3$ synapses are highly stable and less influenced by ASD-related genetic mutations.

Identification of both common and distinct synaptic phenotypes from multiple ASD mouse models may further extend our understanding of the pathophysiology of ASD. In this study, we detected an enhanced turnover of shaft gephyrin clusters in patDp/ + mice but not in NLG R451C mice. The imbalance between excitatory and inhibitory synaptic inputs has been proposed to contribute to the pathophysiology of $\mathrm{ASD}^{8,57}$. NLG R451C mice show an increase in spontaneous inhibitory synaptic events ${ }^{15}$. Conditional $\mathrm{KO}$ of Mecp2 in inhibitory neurons induced a reduction in inhibitory signalling and autism-like stereotypies 9 . Mice with mutant Scnla gene, which is responsible for Dravet's syndrome, show autistic-like behaviour and impairment in GABAergic transmission ${ }^{11}$. These studies indicate that both 
upregulation and suppression in inhibitory synaptic transmission are associated with autistic-like behaviour in model mice. Activity of interneurons should be regulated by multiple factors, including the average level of local circuit activity, feed-forward and feedback mechanisms of information processing, synchronization and oscillation of neuronal populations and neuromodulatory signals. The level of inhibitory synaptic transmission is likely to be regulated by the integration of multiple factors altered in the cortex of ASD mouse models. Further characterization of neuronal connectivity via inhibitory synapses in the process of early cortical development may provide more information on the role of inhibitory synapses in the pathophysiology of ASD.

In addition to alterations in synapse remodelling, we found disruption in the effect of shaft gephyrin clusters on the stability of nearby spines in ASD mouse models. In vivo imaging of both dendritic spines and gephyrin clusters indicated that remodelling of spines and inhibitory shaft synapses is spatially clustered and monocular deprivation increased the frequency of clustering events ${ }^{58}$. These results indicate that the spatial relationship between synapses plays an important role in experiencedependent remodelling of cortical neuron connectivity. The negative effects of shaft inhibitory synapses on nearby spines may be important in the regulation of a clustered formation of spines. Clustering of new spines and inhibitory shaft synapses in the visual cortex may be explained by assuming that the positions of shaft inhibitory synapses determine the hotspots of synapse remodelling, where both new spines and new inhibitory synapses are more likely to be formed. Disruption of local interactions between shaft inhibitory synapses and nearby spines in ASD mouse models may have important consequences in local dendritic integration of synaptic inputs ${ }^{59}$. Impairment in experience-dependent regulation of spine dynamics we found in ASD mouse models may be explained by disorganized integration of sensory inputs to layer $2 / 3$ pyramidal neurons, which showed reduced expression of immediate early gene c-fos in response to acute sensory stimulation. A recent analysis of ensemble activity in the SSC of Fmrl KO mice indicated an abnormally high synchrony of network activity ${ }^{60}$. Further physiological investigations are required to test whether impaired connectivity and imbalance in local excitatory/inhibitory circuits underlie functional deficits in ASD mouse models.

\footnotetext{
Methods

Animals and constructs. All experimental procedures were performed in compliance with the institutional guidelines of the University of Tokyo. Male or female wild-type ICR (Fig. 1) and C57Bl/6J (Fig. 2) mice (Japan SLC, Inc.) were used for in vivo imaging (see Supplementary Tables 1-7 for the number and ages of animals in each experiment). The following ASD mouse models were used in this study: $\mathrm{patDp} /+$ mice with paternal duplication of chromosome $7 \mathrm{c}^{24}$, NLG R451C mice with a point mutation corresponding to the human NLGN-3 R451C ${ }^{15}$ and BTBR $\mathrm{T}+\mathrm{tf}$ mice $^{43}$. Constructs of PSD-95-GFP were previously described ${ }^{34,61}$. An expression plasmid of DsRed2 was constructed by replacing the cDNA sequence from pDsRed2-N1 (Clontech) with GFP of pAct-GFP. An expression plasmid for gephyrin-GFP was constructed by inserting the gephyrin-GFP fragment ${ }^{62}$ into the pCAGGS vector. PSD-95-GFP and DsRed2 were expressed under the control of the $\beta$-actin promoter, and gephyrin-GFP was expressed under the control of the CAG promoter.
}

In utero electroporation. Progenitor cells of layer 2/3 neurons in the SSC and AFC were transfected using in utero electroporation ${ }^{63,64}$. E15.5 timed pregnant mice were deeply anaesthetized with an intraperitoneal injection of pentobarbital $\left(100 \mathrm{mg} \mathrm{kg}^{-1}\right)$ and the uterine horns were exposed. Approximately $1 \mu \mathrm{l}$ of DNA solution (a mixture of DsRed2 plasmid $\left(1 \mu \mathrm{g} \mu \mathrm{l}^{-1}\right)$ with either a PSD-95-GFP plasmid $\left(1-2 \mu \mathrm{g}^{-1}\right)$ or a gephyrin-GFP plasmid $\left.\left(0.1 \mu \mathrm{g} \mu \mathrm{l}^{-1}\right)\right)$ was pressure injected into the lateral ventricle of each embryo through a pulled-glass micropipette. The head of each embryo was placed between tweezer-type electrodes (CUY650P5, NEPA Gene). Square electric pulses $(28-33 \mathrm{~V}, 50 \mathrm{~ms})$ were passed four times at $1 \mathrm{~Hz}$ using an electroporator (CUY21, NEPA Gene).
Surgery. We applied the thinned-skull technique $31,32,65$ to most of the in vivo twophoton imaging. Thinned-skull windows were prepared by carefully thinning a small circular area of the cranium by micro surgical blades to $\sim 15 \mu \mathrm{m}$ thickness Mice expressing fluorescent proteins at postnatal weeks 2-8 were anaesthetized with intraperitoneal injections of ketamine (100 $\mathrm{mg}$ per $\mathrm{kg}$ body weight) and xylazine (10 mg per kg body weight). For young mice, the dose of anaesthesia was reduced to half. Minimum doses of ketamine and xylazine were arbitrarily administered as supplemental anaesthesia. The skull was exposed by incising a midline of the scalp. A small metal pipe (diameter, $3 \mathrm{~mm}$ ) was glued to the skull with dental cement (Sunmedical). Mice were held on a stereotaxic instrument using the pipe. The surface compact bone and the intermediate spongy bone were removed with the help of a spherical drill bit (Fine Science Tools) attached to a hand-held high-speed drill until the thickness of the remaining bone reached $\sim 50 \mu \mathrm{m}$. Further thinning was manually performed using micro surgical blades (Nordland Blade, Salvin Dental) until that part of the skull was $\sim 15 \mu \mathrm{m}$ in thickness. Care was taken to not push the skull during the thinning process to avoid brain damage. The imaging locations were as follows: SSC, bregma $-1.0 /-1.5 \mathrm{~mm}$ and lateral 1.5-2.5/2.0-3.0 $\mathrm{mm}$ (young/adult); AFC, bregma 2.1-3.0 $\mathrm{mm}$ and lateral $0.8-1.4 \mathrm{~mm}$ (Supplementary Fig. 4); barrel cortex, bregma -0.5 to $-1.0 \mathrm{~mm}$ and lateral -3.0 to $-3.5 \mathrm{~mm}$. After in vivo imaging of sensory-manipulated animals, we injected rhodamine dye into the imaging area, prepared tangential brain sections and confirmed that extensive overlap of the imaging areas with the barrel cortex.

The thinned-skull technique cannot be applied to mice younger than PND 20. In young animals, deterioration of the point-spread function through the thinnedskull window was severe, probably because of the immature spongy bone tissue of the cranium. As the collection of synaptic dynamics data in young animals was necessary in some sets of experiments (Figs 1 and $4 \mathrm{e}-\mathrm{h}$ ), we switched to the openskull window technique in these cases $^{33}$. Our comparisons of the two techniques indicated that the open-skull window technique can reliably report synaptic dynamics when great care was taken to prevent the activation of glia in the surgical area. The surgical procedures for open-skull windows are as follows. A circular groove was made on the skull with a trephine attached to a high-speed drill. The central island of the skull bone was carefully lifted using a microprobe and forceps with the underlying dura intact. The cranial window was covered with a sterile 3-mm coverglass and sealed with dental cement. We evaluated the surgical procedures of individual experimenters by scoring multiple parameters, including the total operation time, bleeding from the bone tissue, brain swelling, intact brain surface after bone removal, bleeding from the dura and cleanliness of the glass window after application of the adhesive. Only if all the scores were above 4 (very good), the surgery was judged as successful. We routinely checked the extent of glial activation after the open-skull surgery and found no sign of increase in Iba-1 immunoreactivity, indicating little activation of microglia (Supplementary Fig. 8). More importantly, the spine turnover rate at postnatal week 8 measured by using the open-skull window technique was $3.61 \%$ (Fig. 1f), which was in the range of spine turnover rates previously reported using the thinned-skull technique ${ }^{66}$. We further compared spine turnover rates with two types of cranial windows at postnatal week 3 and found no significant differences (Supplementary Fig. 9a). The first imaging session was just after surgery of open-skull window in this study, and acute effects of the surgery may affect spine dynamics. However, sequential imaging for 3 consecutive days after preparation of open-skull windows revealed no immediate effect of invasive procedures (Supplementary Fig. 9b). From these considerations, we concluded that the dynamic properties measured through the open-skull windows reliably reported the turnover of native synapses in vivo.

In vivo imaging. After surgery, mice were immediately placed under a scanning microscope (FV-300; Olympus) equipped with a pulsed laser (MaiTai HP, Spectra Physics) and a $\times 25$ water immersion objective lens (XLPlan N, numerical aperture (NA) 1.05, Olympus). To concurrently obtain GFP and DsRed2 signals, the wavelength was set to $920 \mathrm{~nm}$, and the average power of the laser after the objective lens was $\sim 20 \mathrm{~mW}$. GFP and DsRed2 fluorescence signals were separated with a dichroic mirror $(550 \mathrm{~nm})$ and barrier filters $(515 / 30 ; 575 \mathrm{IF})$. The imaging area was $565 \times 565 \mu \mathrm{m}$ (low magnification) or $78 \times 78 \mu \mathrm{m}$ (high magnification), and the step size of the $z$ stack was set to 2 or $0.75 \mu \mathrm{m}$. The pixel sizes of single horizonta images were set to $512 \times 512$. Imaging depth was $<100 \mu \mathrm{m}$ from the pia. Apical dendritic branches in layer $2 / 3$ pyramidal neurons were randomly selected for analyses. We recorded the brain vasculature pattern using a charge-coupled device camera (GZ-MG70, Victor) to create vasculature maps, which facilitated the identification of the imaged area in previous sessions. After an imaging session, the scalp of mice with thinned-skull windows was sutured. Mice before weaning (younger than postnatal week 2) were housed with their littermates and mothers between imaging sessions. Mice after weaning (older than postnatal week 3 ) were individually housed between imaging sessions. Intervals of in vivo imaging for the comparison of dynamics at different time points of postnatal development (Fig. 1c,e,f) were set as 1 day. Developmental decline of spine turnover could be better illustrated with this imaging condition. Intervals of in vivo imaging for the comparison of wild-type and ASD mouse models at postnatal week 3 were set as 2 days (Figs 3-5). With longer time intervals, the rates of gain and loss of spines could be more reliably calculated, mainly because of the increased numbers of added/lost spines in a given pair of image stacks taken at two time points. 
Sensory manipulations. For imaging studies, whiskers were deprived in a chessboard pattern ${ }^{44}$ by a pair of tweezers after the first imaging session. For c-fos immunostaining, mice were anaesthetized with isoflurane and all whiskers, except row $\mathrm{C}$, were deprived by a pair of tweezers. After recovery of anaesthesia, mice were exposed to the enriched environment for $1 \mathrm{~h}$ (ref. 46).

Immunohistochemistry. Mice at postnatal week 3 were anaesthetized and perfused with $4 \%$ paraformaldehyde. The brains were dissected and cut into $50-\mu \mathrm{m}-$ thick slices using a vibratome. Brain sections were treated with $0.5 \%$ Triton X-100 in PBS, blocked with 5\% NGS and then reacted with primary antibodies, followed by secondary antibodies. The antibodies used in this study were as follows: guinea pig anti-VGluT2 (1:400, Millipore), rabbit anti-VGluT1 $(1: 400)^{67}$, rabbit anti-Iba-1 (1:1,000, WAKO), mouse anti-gephyrin (1:1,000, Synaptic Systems), rabbit anti-cfos (1:5,000, Calbiochem), Alexa 647-labelled goat anti-mouse IgG (1:500, Invitrogen), Alexa 647-labelled goat anti-guinea pig IgG (1:600, Invitrogen) and Alexa 488- or 647-labelled goat anti-rabbit IgG (1:500, Invitrogen). Images were obtained from layer 1 of the SSC using an FV1000 confocal laser-scanning microscope (Olympus) with a $\times 60$ oil-immersion lens (Olympus, PlanApoN, NA 1.42 ) or a $\times 20$ lens (Olympus, UPlanApo, NA 0.70).

Data analysis. Image processing and analysis were performed using Image software (NIH, http://rsb.info.nih.gov/ij). All analyses were performed using raw three-dimensional stack images. The same dendritic segments were identified from the three-dimensional stack images at different time points. We classified dendritic protrusions into either spines or filopodia. The filopodia were defined as long, thin structures without apparent spine heads and had not accumulated PSD-95-GFP. PSD-95-GFP- $(-)$ spines had small spine heads (Fig. 1d) and were shorter than typical filopodia. Furthermore, the survival rate of PSD-95-GFP- $(-)$ spines over 1 day in 2-week-old mice was higher than that of filopodia (PSD-95- $(-)$ spines, $50.0 \%$; filopodia, 20.8\%), indicating that PSD-95-GFP-( - ) spines had properties different from those of filopodia. Spines were considered identical between the two images if they were within $0.5 \mu \mathrm{m}$ of their expected positions, based on their spatial relationship to adjacent spines or other fiducial marks such as dendritic branch points. Newly formed spines were those identified only at the second time point. Eliminated spines were those that existed only at the first time point but disappeared at the second time point. Spines present at both time points were categorized as stable spines. As two-photon microscopy has lower resolution in the $z$ axis, only dendritic protrusions that were clearly projecting laterally were included in the analysis ${ }^{68}$.

For the classification of spines with or without PSD-95-GFP clusters, we defined the clustering index $(\mathrm{CI})$ as follows:

$$
C I=\frac{G_{\text {spine }} / R_{\text {spine }}}{G_{\text {shaft }} / R_{\text {shaft }}}
$$

$G_{\text {spine }}$ and $R_{\text {spine }}$ were the average intensities of either PSD-95-GFP ( $G_{\text {spine }}$ ) or DsRed2 $\left(R_{\text {spine }}\right)$ fluorescence from boxed regions of interest (ROIs) $(0.46 \times 0.46 \mu \mathrm{m})$ positioned at the centre of spine heads in a single plane of the $z$-stack images, where the intensity of the spine DsRed 2 signal was highest and the spines were well separated from other fluorescent structures in the vicinity. ROIs were placed at the distal part of thin or stubby spines if they were without clear heads. $G_{\text {shaft }}$ and $R_{\text {shaft }}$ were the average intensities measured with an identical setting of ROIs but with their positions on nearby dendritic shafts. In some cases, $G_{\text {shaft }}$ was not high enough for accurate measurement and was substituted by the average of multiple $G_{\text {shaft }}$ values from the ROIs placed on dendrites with sufficient GFP signals and closest to the ROIs on spines. We sampled 1,454 different dendritic positions for the calculation of $G_{\text {shaft }} / R_{\text {shaft }}$ and found that $95 \%$ of individual $G_{\text {shaft }} / R_{\text {shaft }}$ values were within $50-200 \%$ of their average. Therefore, the threshold for the CI of PSD-95-GFP- $(+)$ spines was set at 2.0 (Supplementary Fig. 10a,b). Spines with their $G_{\text {spine }} / R_{\text {spine }}$ values more than twice the $G_{\text {shaft }} / R_{\text {shaft }}$ values were classified as spines containing PSD-95-GFP clusters.

For detection of gephyrin-GFP clusters, we first measured basal GFP intensities on the dendritic shafts $\left(\mathrm{GFP}_{\text {shaft }}\right)$ from ten ROIs $(0.46 \times 0.46 \mu \mathrm{m})$ placed on the dendritic areas without gephyrin clusters. Pixels containing GFP fluorescence intensity more than twofold of the $\mathrm{GFP}_{\text {shaft }}$ were isolated by thresholding, and binary images were created. Clustered pixels with their area more than $0.28 \mu \mathrm{m}^{2}$ in binary images were further isolated, and clusters with their average intensities more than twice the values of nearby dendritic regions were selected as gephyrin clusters. Gephyrin clusters were judged to be identical with criteria similar to the scoring of identical spines at different time points. Gephyrin-GFP- $(+)$ spines were defined as the spines containing gephyrin-GFP clusters at the first or second imaging sessions. Gephyrin-GFP- $(-)$ spines were the remaining spines without gephyrin-GFP clusters in both imaging sessions.

In this study, we provided two types of parameters for the evaluation of spine dynamics. The first parameter was gain/loss of spines per unit length of dendrites. The second parameter was the turnover rate expressed as a percentage of newly formed and/or eliminated spines per total spines. To calculate these two types of parameters, we first measured the following values:

$L_{\text {dendrite: }}$ length of dendritic segments analysed

$N_{\text {total1 }}$ and $N_{\text {total2 }}$ : total number of spines in the first and second imaging sessions
$N_{\text {gain }}$ and $N_{\text {loss }}$ : number of appeared and disappeared spines between two imaging sessions

When spines were classified by either PSD-95-GFP or gephyrin-GFP, the above values were measured within each subset of classified spines. Using these values, the following parameters were calculated:

$$
\text { Gain of spines per } \mu \mathrm{m}=\frac{N_{\text {gain }}}{L_{\text {dendrite }}}
$$

(Figs 1f, 2g, 3d,h, 4d,h, 5d,h and 7b,c, and Supplementary Figs 1a, 5b and 9b)

$$
\text { Loss of spines per } \mu \mathrm{m}=\frac{N_{\text {loss }}}{L_{\text {dendrite }}}
$$

(Figs 1f, 2g, 3d,h, 4d,h, 5d,h and 7b,c, and Supplementary Figs 1a, 5b and 9b)

$$
\text { Turnover rate }(\%) \text { of total spines }=\frac{N_{\text {gain }}+N_{\text {loss }}}{N_{\text {total1 }}+N_{\text {total } 2}}
$$

(Figs 3c,g, 4c,g and 5c,g, and Supplementary Fig. 5a)

$$
\text { Turnover rate }(\%) \text { of spine gain }=\frac{N_{\text {gain }}}{\left(N_{\text {total1 }}+N_{\text {total2 } 2) / 2}\right.}
$$

(Fig. 1c, and Supplementary Figs 6a and 9a)

$$
\text { Turnover rate }(\%) \text { of spine loss }=\frac{N_{\text {loss }}}{\left(N_{\text {total1 }}+N_{\text {total2 } 2}\right) / 2}
$$

(Fig. 1c, and Supplementary Figs 6a and 9a)

Gain and loss of gephyrin clusters in Fig. 6d,e,g,h were calculated using the formula similar to equations (2) and (3), with substitution of $N_{\text {gain }}$ and $N_{\text {loss }}$ by the number of appeared and disappeared gephyrin clusters between two imaging sessions, respectively.

To identify the transition between PSD-95-GFP- $(+)$ and $(-)$ spines, we calculated CIs of the individual spines at two time points using equation (1) and plotted them in the $x-y$ space (Supplementary Fig. 10d). As we set the threshold of PSD-95-GFP clustering at $C I$ as 2.0, new spines were categorized as PSD-positive if their CIs were higher than 2.0. Therefore, we defined the transition from PSDnegative to PSD-positive spines only when their CI changed to $>2.0$. The following equation defined the area in the $x-y$ space where transition from PSD-negative to PSD-positive spines occurred:

$$
C I_{2 \text { nd }} \geq C I_{1 s t}+2.0 \text { and } C I_{1 s t}<2.0
$$

$C_{1 s t}$ and $C_{2 \text { nd }}$ are CIs at the first and second imaging sessions. The transition from PSD-positive to PSD-negative spines was also defined by the following equation:

$$
C I_{1 s t} \geq C I_{2 \text { nd }}+2.0 \text { and } C I_{2 \text { nd }}<2.0
$$

The remaining spines were judged as PSD-negative when their CIs did not change to $>2.0$ and their average CIs were $<2.0$. These conditions can be described by the following equation:

$$
\left|C I_{1 s t}-C I_{2 n d}\right|<2.0 \text { and } \frac{\left(C I_{1 s t}+C I_{2 n d}\right)}{2}<2.0
$$

The areas defined by equations (7), (8) and (9) are the areas filled with different colours in Supplementary Fig. 10b. The remaining area in this graph defined the spines that remained PSD-positive at two time points. Spines with large spine heads (spines with large $R_{\text {spine }}$ ) may be erroneously categorized as PSD-95-negative spines. To evaluate this possibility, we randomly selected large persistent spines $(n=30)$ at postnatal week 8 and plotted their CIs (Supplementary Fig. 10c). Most of these stable spines in the mature cortex should have synaptic connections, and our protocol successfully categorized them as PSD-95-positive spines. By this protocol, CIs of all spines from two successive imaging sessions could be plotted in the $x-y$ space, and they could be automatically classified into four types of dynamic spines and four types of persistent spines (Supplementary Fig. 10d). Importantly, the manual classification of spines into the same categories by multiple investigators confirmed the validity of automatic categorization.

For measurement of spine volume, ROIs were set to cover the entire spine heads, and the integrated intensities of single spines in a single plane were quantified. Integrated intensities were normalized to the mean intensity of adjacent dendrites measured from boxed ROIs $(0.75 \times 0.75 \mu \mathrm{m})$. Backgrounds were subtracted before normalization.

For the analysis of apposition between dendritic spines and VGluT1 (or VGluT2) immunopositive puncta, we first selected dendritic spines from confocal images of DsRed2-filled dendrites. Subsequently, GFP images were overlaid with DsRed 2 images that aided in judging the presence or absence of gephyrin clusters for each spine. Another investigator then manually analysed the juxtapositon between dendritic spines and VGluT1 (or VGluT2) puncta in a blind manner without referring to the GFP images. When pixel areas corresponding to spine heads were contacted or overlapped with pixel areas judged positive with VGluT1 (or VGluT2) signals, the spines were scored to have apposition to VGluT1 (or VGluT2) puncta. To estimate the overlap at chance level, we prepared image stacks with a GFP channel shifted by 50 pixels $(\sim 5 \mu \mathrm{m})$ in both $x$ and $y$ directions, and performed the same analysis.

For the analysis of c-fos immunoreactive cells, binary images were created by setting the threshold value to the mean +3 s.d. of the background signal. 
The borders of activated barrel columns were identified by using VGluT2 immunoractivity, which was strong in the central area of barrel columns. The number of c-fos immunoreactive cells in layer $2 / 3$ and layer 4 of activated columns was counted by using particle analysis plugin of ImageJ, and densities of c-fos immunoreactive cells were calculated.

For statistical analysis, data are presented as means \pm s.e.m. Paired means were compared using $t$-tests. Multiple means were compared using one-way analysis of variance.

\section{References}

1. Geschwind, D. H. Advances in autism. Annu. Rev. Med. 60, 367-380 (2009)

2. Amaral, D. G., Schumann, C. M. \& Nordahl, C. W. Neuroanatomy of autism. Trends. Neurosci. 31, 137-145 (2008).

3. Courchesne, E. \& Pierce, K. Why the frontal cortex in autism might be talking only to itself: local over-connectivity but long-distance disconnection. Curr. Opin. Neurobiol. 15, 225-230 (2005).

4. Zoghbi, H. Y. Postnatal neurodevelopmental disorders: meeting at the synapse? Science 302, 826-830 (2003).

5. Penzes, P., Cahill, M. E., Jones, K. A., VanLeeuwen, J. E. \& Woolfrey, K. M Dendritic spine pathology in neuropsychiatric disorders. Nat. Neurosci. 14, 285-293 (2011).

6. Geschwind, D. H. Autism: many genes, common pathways? Cell 135, 391-395 (2008).

7. State, M. W. \& Levitt, P. The conundrums of understanding genetic risks for autism spectrum disorders. Nat. Neurosci. 14, 1499-1506 (2011).

8. Südhof, T. C. Neuroligins and neurexins link synaptic function to cognitive disease. Nature 455, 903-911 (2008).

9. Chao, H. T. et al. Dysfunction in GABA signalling mediates autism-like stereotypies and Rett syndrome phenotypes. Nature 468, 263-269 (2010).

10. Auerbach, B. D., Osterweil, E. K. \& Bear, M. F. Mutations causing syndromic autism define an axis of synaptic pathophysiology. Nature 480, 63-68 (2011).

11. Han, S. et al. Autistic-like behaviour in Scnla $+/-$ mice and rescue by enhanced GABA-mediated neurotransmission. Nature 489, 385-390 (2012).

12. Baudouin, S. J. et al. Shared synaptic pathophysiology in syndromic and nonsyndromic rodent models of autism. Science 338, 128-132 (2012).

13. Bangash, M. A. et al. Enhanced polyubiquitination of Shank3 and NMDA receptor in a mouse model of autism. Cell 145, 758-772 (2011)

14. Peca, J. et al. Shank3 mutant mice display autistic-like behaviours and striatal dysfunction. Nature 472, 437-442 (2011).

15. Tabuchi, K. et al. A neuroligin-3 mutation implicated in autism increases inhibitorysynaptic transmission in mice. Science 318, 71-76 (2007).

16. Guy, J., Hendrich, B., Holmes, M., Martin, J. E. \& Bird, A. A mouse Mecp2-null mutation causes neurological symptoms that mimic Rett syndrome. Nat. Genet. 27, 322-326 (2001)

17. Chen, R. Z., Akbarian, S., Tudor, M. \& Jaenisch, R. Deficiency of methyl-CpG binding protein-2 in CNS neurons results in a Rett-like phenotype in mice. Nat. Genet. 27, 327-331 (2001)

18. Goorden, S. M., van Woerden, G. M., van der Weerd, L., Cheadle, J. P. \& Elgersma, Y. Cognitive deficits in Tscl $+/$ - mice in the absence of cerebral lesions and seizures. Ann. Neurol. 62, 648-655 (2007).

19. Mineur, Y. S., Huynh, L. X. \& Crusio, W. E. Social behavior deficits in the Fmr1 mutant mouse. Behav. Brain Res. 168, 172-175 (2006)

20. Michalon, A. et al. Chronic pharmacological mGlu5 inhibition corrects fragile $\mathrm{X}$ in adult mice. Neuron 74, 49-56 (2012).

21. Sato, A. et al. Rapamycin reverses impaired social interaction in mouse models of tuberous sclerosis complex. Nat. Commun. 3, 1292 (2012).

22. Lang, M. et al. Selective preservation of MeCP2 in catecholaminergic cells is sufficient to improve the behavioral phenotype of male and female Mecp2deficient mice. Hum. Mol. Genet. 22, 358-371 (2013).

23. Abrahams, B. S. \& Geschwind, D. H. Advances in autism genetics: on the threshold of a new neurobiology. Nat. Rev. Genet. 9, 341-355 (2008).

24. Nakatani, J. et al. Abnormal behavior in a chromosome-engineered mouse model for human 15q11-13 duplication seen in autism. Cell 137, 1235-1246 (2009).

25. Fox, K. Anatomical pathways and molecular mechanisms for plasticity in the barrel cortex. Neuroscience 111, 799-814 (2002)

26. Feldman, D. E. \& Brecht, M. Map plasticity in somatosensory cortex. Science 310, 810-815 (2005)

27. Douglas, R., Markram, H. \& Martin, K. in The Synaptic Organization of the Brain (ed. Shepherd, G. M.) 499-558 (Oxford University Press, 2004).

28. Miller, M. Maturation of rat visual cortex. I. A quantitative study of Golgi-impregnated pyramidal neurons. J. Neurocytol. 10, 859-878 (1981).

29. De Felipe, J., Marco, P., Fairén, A. \& Jones, E. G. Inhibitory synaptogenesis in mouse somatosensory cortex. Cereb. Cortex 7, 619-634 (1997).

30. Ashby, M. C. \& Isaac, J. T. Maturation of a recurrent excitatory neocortical circuit by experience-dependent unsilencing of newly formed dendritic spines. Neuron 70, 510-521 (2011)
31. Zuo, Y., Lin, A., Chang, P. \& Gan, W. B. Development of long-term dendritic spine stability in diverse regions of cerebral cortex. Neuron 46, 181-189 (2005).

32. Zuo, Y., Yang, G., Kwon, E. \& Gan, W. B. Long-term sensory deprivation prevents dendritic spine loss in primary somatosensory cortex. Nature 436, 261-265 (2005)

33. Holtmaat, A. et al. Long-term, high-resolution imaging in the mouse neocortex through a chronic cranial window. Nat. Protoc. 4, 1128-1144 (2009).

34. Okabe, S., Kim, H. D., Miwa, A., Kuriu, T. \& Okado, H. Continual remodeling of postsynaptic density and its regulation by synaptic activity. Nat. Neurosci. 2, 804-811 (1999).

35. Okabe, S., Miwa, A. \& Okado, H. Spine formation and correlated assembly of presynaptic and postsynaptic molecules. J. Neurosci. 21, 6105-6114 (2001).

36. Gray, N. W., Weimer, R. M., Bureau, I. \& Svoboda, K. Rapid redistribution of synaptic PSD-95 in the neocortex in vivo. PLoS Biol. 4, e370 (2006).

37. Kubota, Y., Hatada, S., Kondo, S., Karube, F. \& Kawaguchi, Y. Neocortical inhibitory erminals innervate dendritic spines targeted by thalamocortical afferents. J Neurosci. 31, 1139-1150 (2007).

38. Courchesne, E. et al. Mapping early brain development in autism. Neuron 56, 399-413 (2007)

39. Qiu, S., Anderson, C. T., Levitt, P. \& Shepherd, G. M. Circuit-specific intracortical hyperconnectivity in mice with deletion of the autism-associated Met receptor tyrosine kinase. J. Neurosci. 31, 5855-5864 (2011).

40. Mitchell, B. D. \& Macklis, J. D. Large-scale maintenance of dual projections by callosal and frontal cortical projection neurons in adult mice. J. Comp. Neurol. 482, 17-32 (2005).

41. Tennant, K. A. et al. The organization of the forelimb representation of the C57BL/6 mouse motor cortex as defined by intracortical microstimulation and cytoarchitecture. Cereb. Cortex 21, 865-876 (2011).

42. Van De Werd, H. J., Rajkowska, G., Evers, P. \& Uylings, H. B. Cytoarchitectonic and chemoarchitectonic characterization of the prefrontal cortical areas in the mouse. Brain Struct. Funct. 214, 339-353 (2010).

43. McFarlane, H. G. et al. Autism-like behavioral phenotypes in BTBR T $+\mathrm{tf} / \mathrm{J}$ mice. Genes Brain Behav. 7, 152-163 (2008).

44. Holtmaat, A., Wilbrecht, L., Knott, G. W., Welker, E. \& Svoboda, K. Experience-dependent and cell-type-specific spine growth in the neocortex. Nature 441, 979-983 (2006).

45. Pan, F., Aldridge, G. M., Greenough, W. T. \& Gan, W. B. Dendritic spine instability and insensitivity to modulation by sensory experience in a mouse model of fragile X syndrome. Proc. Natl Acad. Sci. USA 107, 17768-17773 (2010).

46. Bisler, S. et al. Expression of c-Fos, ICER, Krox-24 and JunB in the whisker-tobarrel pathway of rats: time course of induction upon whisker stimulation by tactile exploration of an enriched environment. J. Chem. Neuroanat. 23, 187-198 (2002).

47. Budreck, E. C. \& Scheiffele, P. Neuroligin-3 is a neuronal adhesion protein at GABAergic and glutamatergic synapses. Eur. J. Neurosci. 26, 1738-1748 (2007).

48. Cruz-Martin, A., Crespo, M. \& Portera-Cailliau, C. Delayed stabilization of dendritic spines in fragile X mice. J. Neurosci. 30, 7793-7803 (2010).

49. Yuste, R. \& Bonhoeffer, T. Genesis of dendritic spines: insights from ultrastructural and imaging studies. Nat. Rev. Neurosci. 5, 24-34 (2004).

50. Yasumatsu, N., Matsuzaki, M., Miyazaki, T., Noguchi, J. \& Kasai, H. Principles of long-term dynamics of dendritic spines. J. Neurosci. 28, 13592-13608 (2008)

51. Hill, T. C. \& Zito, K. LTP-induced long-term stabilization of individual nascent dendritic spines. J. Neurosci. 33, 678-686 (2013).

52. Etherton, M. et al. Autism-linked neuroligin-3 R451C mutation differentially alters hippocampal and cortical synaptic function. Proc. Natl Acad. Sci. USA 16, 13764-13769 (2011).

53. Feldman, D. E., Nicoll, R. A. \& Malenka, R. C. Synaptic plasticity at thalamocortical synapses in developing rat somatosensory cortex: LTP, LTD, and silent synapses. J. Neurobiol. 41, 92-101 (1999).

54. Crair, M. C. \& Malenka, R. C. A critical period for long-term potentiation at thalamocortical synapses. Nature 375, 325-328 (1995).

55. Lu, S. M. \& Lin, R. C. Thalamic afferents of the rat barrel cortex: a light- and electron-microscopic study using Phaseolus vulgaris leucoagglutinin as an anterograde tracer. Somatosens. Mot. Res. 10, 1-16 (1993).

56. Yu, C., Derdikman, D., Haidarliu, S. \& Ahissar, E. Parallel thalamic pathways for whisking and touch signals in the rat. PLoS Biol. 4, e124 (2006)

57. Rubenstein, J. L. \& Merzenich, M. M. Model of autism: increased ratio of excitation/inhibition in key neural systems. Genes Brain Behav. 2, 255-267 (2003).

58. Chen, J. L. et al. Clustered dynamics of inhibitory synapses and dendritic spines in the adult neocortex. Neuron 74, 361-373 (2012).

59. Spruston, N. Pyramidal neurons: dendritic structure and synaptic integration. Nat. Rev. Neurosci. 9, 206-221 (2008). 
60. Gonçalves, J. T., Anstey, J. E., Golshani, P. \& Portera-Cailliau, C. Circuit level defects in the developing neocortex of Fragile X mice. Nat. Neurosci. 16, 903-909 (2013).

61. Ebihara, T., Kawabata, I., Usui, S., Sobue, K. \& Okabe, S. Synchronized formation and remodeling of postsynaptic densities: long-term visualization of hippocampal neurons expressing postsynaptic density proteins tagged with green fluorescent protein. J. Neurosci. 23, 2170-2181 (2003).

62. Kuriu, T., Yanagawa, Y. \& Konishi, S. Activity-dependent coordinated mobility of hippocampal inhibitory synapses visualized with presynaptic and postsynaptic tagged-molecular markers. Mol. Cell. Neurosci. 49, 184-195 (2012).

63. Niwa, M. et al. Knockdown of DISC1 by in utero gene transfer disturbs postnatal dopaminergic maturation in the frontal cortex and leads to adult behavioral deficits. Neuron 65, 480-489 (2010).

64. Tabata, H. \& Nakajima, K. Efficient in utero gene transfer system to the developing mouse brain using electroporation: visualization of neuronal migration in the developing cortex. Neuroscience 103, 865-872 (2001).

65. Yang, G., Pan, F., Parkhurst, C. N., Grutzendler, J. \& Gan, W. B. Thinned-skull cranial window technique for long-term imaging of the cortex in live mice. Nat. Protoc. 5, 201-208 (2010).

66. Xu, H. T., Pan, F., Yang, G. \& Gan, W. B. Choice of cranial window type for in vivo imaging affects dendritic spine turnover in the cortex. Nat. Neurosci. 10, 549-551 (2007).

67. Takamori, S., Rhee, J. S., Rosenmund, C. \& Jahn, R. Identification of a vesicular glutamate transporter that defines a glutamatergic phenotype in neurons. Nature 407, 189-194 (2000).

68. Kondo, S., Kohsaka, S. \& Okabe, S. Long-term changes of spine dynamics and microglia after transient peripheral immune response triggered by LPS in vivo. Mol. Brain 4, 27 (2011)

\section{Acknowledgements}

We thank Taisuke Mizuguchi and Noriyuki Hayashi for data analysis, Satoe Ebihara for technical support, and Dr Shigeo Takamori for providing VGluT1 antibody. The BTBR $\mathrm{T}+\mathrm{tf}$ mouse strain (RBRC 01206) was provided by RIKEN BRC through the National Bio-Resource Project of the MEXT, Japan. This work was supported by Grants-in-Aid for Scientific Research $(21220008,25117006$ and 26250014 to S.O.), 'Development of biomarker candidates for social behavior' study conducted under the Strategic Research

Program for Brain Sciences by the Ministry of Education, Culture, Sports, Science and Technology of Japan (S.O.), Global COE Program (Integrative Life Science Based on the Study of Biosignaling Mechanisms to S.O.), Grant-in-Aid for JSPS Fellows (11J05614 to M.I.), Takeda Science Foundation (S.O.) and Naito Memorial Foundation (S.O.).

\section{Author contributions}

M.I. and S.T. designed and performed the experiments, analysed the data and wrote the manuscript. T.K. helped with molecular biology. K.T. and T.T. helped with mouse molecular genetics. S.O designed experiments and wrote the manuscript.

\section{Additional information}

Supplementary Information accompanies this paper at http://www.nature.com/ naturecommunications

Competing financial interests: The authors declare no competing financial interests.

Reprints and permission information is available online at http://npg.nature.com/ reprintsandpermissions/

How to cite this article: Isshiki, M. et al. Enhanced synapse remodelling as a common phenotype in mouse models of autism. Nat. Commun. 5:4742 doi: 10.1038/ncomms5742 (2014) 